\title{
Guideline for stratified screening and surveillance in patients with high risk of primary liver cancer (2020)
}

\author{
Huiguo Ding ${ }^{1}$, Hong $\mathrm{Tu}^{2}$, Chunfeng $\mathrm{Qu}^{3}$, Guangwen $\mathrm{Cao}^{4}$, Hui Zhuang ${ }^{5}$, Ping Zhao ${ }^{6}$, Xiaoyuan $\mathrm{Xu}^{7}$, \\ Yongping Yang ${ }^{8}$, Shichun Lu'; Committee for Prevention and Control of Hepatobiliary and Pancreatic \\ Diseases of Chinese Preventive Medicine Association, Committee of Hepatology of Chinese Research \\ Hospital Association, Hepatology Society of Chinese Medical Association, Prevention of Infection \\ Related Cancer (PIRCA) Group, Specialist Committee of Cancer Prevention and Control of Chinese \\ Preventive Medicine Association
}

\begin{abstract}
'Department of Hepatology and Gastroenterology, Beijing Youan Hospital, Capital Medical University, Beijing 100069, China. ${ }^{2}$ State Key Laboratory of Oncogenes and Related Genes, Shanghai Cancer Institute, Shanghai 200032, China.

${ }^{3}$ State Key Lab of Molecular Oncology, National Cancer Center/National Clinical Research Center for Cancer/Cancer Hospital, Chinese Academy of Medical Sciences and Peking Union Medical College, Beijing 100021, China.

${ }^{4}$ Department of Epidemiology, School of Basic Medicine, The Second Military Medical University, Shanghai 200433, China.

${ }^{5}$ Department of Pathogenic Biology, School of Basic Medicine, Peking University Health Science Center, Beijing 100191, China.

${ }^{6}$ Academic Department of Cancer Foundation of China, Beijing 100021, China.

${ }^{7}$ Department of Infectious Diseases, Peking University First Hospital, Beijing 100034, China.

${ }^{8}$ Department of Liver disease, The Fifth Medical Center of Chinese PLA General Hospital, Beijing 100039, China.

${ }^{9}$ Faculty of Hepato-pancreato-biliary Surgery, Chinese PLA General Hospital, Beijing 100853, China.
\end{abstract}

Correspondence to: Prof. Yongping Yang, Department of liver disease, the Fifth Medical Center of Chinese PLA General Hospital, No.100 Xi Si Huan Zhong Road, Fengtai District, Beijing 100039, China. E-mail: yongpingyang@hotmail.com; Prof. Xiaoyuan Xu, Department of Infectious Diseases, Peking University First Hospital, No. 8 Xishiku Street, Xicheng District, Beijing 100034, E-mail: xiaoyuanxu6@163.com; Prof. Shichun Lu, Faculty of Hepato-pancreato-biliary Surgery, Chinese PLA General Hospital, No. 28 Fuxing Road, Haidian District, Beijing, 100853, China. E-mail: Isc620213@aliyun.com

How to cite this article: Ding H, Tu H, Qu C, Cao G, Zhuang H, Zhao P, Xu X, Yang Y, Lu S, Committee for Prevention and Control of Hepatobiliary and Pancreatic Diseases of Chinese Preventive Medicine Association, Committee of Hepatology of Chinese Research Hospital Association, Hepatology Society of Chinese Medical Association, Prevention of Infection Related Cancer (PIRCA) Group, Specialist Committee of Cancer Prevention and Control of Chinese Preventive Medicine Association. Guideline for stratified screening and surveillance in patients with high risk of primary liver cancer (2020). Hepatoma Res 2021;7:17. http://dx.doi.org/10.20517/2394-5079.2021.13

Received: 4 Feb 2021 Accepted: 4 Feb 2021 Published: 25 Feb 2021

Academic Editor: Guang-Wen Cao Copy Editor: Xi-Jun Chen Production Editor: Xi-Jun Chen

\begin{abstract}
The age-adjusted incidence of primary liver cancer (PLC) has been declining in China. However, PLC cases in China account for $55 \%$ globally. The disease burden is still high and the five-year survival rate has not improved significantly in the past two decades. This guideline outlines PLC screening in populations with high risk, both
\end{abstract}

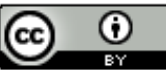

(C) The Author(s) 2021. Open Access This article is licensed under a Creative Commons Attribution 4.0 International License (https://creativecommons.org/licenses/by/4.0/), which permits unrestricted use, sharing, adaptation, distribution and reproduction in any medium or format, for any purpose, even commercially, as long as you give appropriate credit to the original author(s) and the source, provide a link to the Creative Commons license, and indicate if changes were made.
}

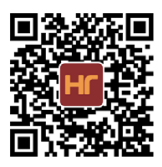


in the hospital and community settings. Liver cirrhosis and chronic hepatitis B are the main causes of PLC in China. For better PLC surveillance and screening in clinical practices, it is recommended that these populations be stratified into four risk levels, namely, low-, intermediate-, high-, and extremely high-risk. A lifelong surveillance is suggested for those with risks of PLC. The intervals and tools for surveillance and screening are recommended based on risk levels. Abdominal ultrasonography combined with serum alpha-fetoprotein tests (routine surveillance) is recommended every 6 months for high risk PLC. Routine surveillance every 3 months and enhanced CT/MRI examinations every 6-12 months is recommended for those with extremely high risk of PLC. The surveillance interval can be extended to one year or longer for those with low or intermediate risk because the annual incidence of low risk PLC is relatively low. The cost-effectiveness of these recommendations remains to be evaluated.

Keywords: Primary liver cancer, cirrhosis, chronic hepatitis B, screening, surveillance

\section{INTRODUCTION}

Primary liver cancer (PLC), referred in this work as liver cancer, includes hepatocellular carcinoma (HCC) originating from hepatocytes, intrahepatic cholangiocarcinoma (ICC) originating from cholangiocytes, and combined hepatocellular cholangiocarcinoma $(\mathrm{CHC})^{[1]}$. Of these, HCC accounts for $85 \%-90 \%$ of all liver cancer. In recent years, great technological progress has been made in the diagnosis and treatment of liver cancer in China, but the age-standardized overall 5-year net survival rate of patients has only increased from $11.7 \%$ between 2000 and 2004 to $14.1 \%$ between 2010 and 2014, without significant improvements ${ }^{[2]}$. However, the 5-year overall survival (OS) rate of 0/A HCC patients staged by the Barcelona clinical staging of liver cancer (BCLC) criteria who received radical treatment ranged from $69.0 \%$ to $86.2 \%$, and the 5 -year OS rate of those patients who underwent surgical resection was $56.9 \%{ }^{[3]}$. Therefore, scientifically defining the high-risk population of liver cancer and formulating a stratified surveillance regimen are mandatory for early detection, early diagnosis, early therapy, and better OS rate of liver cancer.

Differences continue to prevail in the definition of high-risk population of liver cancer and screening surveillance regimen in relevant guidelines for the diagnosis and treatment of liver cancer newly issued by the American Association for the Study of Liver Diseases (AASLD), European Association for the Study of the Liver (EASL), Korean Liver Cancer Association-National Cancer Center Korea, National Comprehensive Cancer Network (NCCN), European Society for Medical Oncology and Asian-Pacific Association for the Study of the Liver (APASL) ${ }^{[4-9]}$. In 2019, National Health Commission (NHC) of China updated the Guidelines for Diagnosis and Treatment of Primary Liver Cancer, which defined patients with chronic viral hepatitis, alcoholic and non-alcoholic hepatitis, and liver cirrhosis due to various causes as a high-risk population for liver cancer, and recommended abdominal ultrasonography (US) combined with serum alpha-fetoprotein (AFP) every 6 months as their surveillance regimen ${ }^{[10]}$. In 2014, the Japanese Society of Hepatology stratified a high-risk population for whom a surveillance regimen composed of AFP, des- $\gamma$-carboxy abnormal prothrombin (DCP), and lens culinaris agglutinin-reactive fraction of AFP (AFP-L3) combined with two of the three imaging tests including US or enhanced CT or MRI every 3-4 months was adopted ${ }^{[11]}$. BCLC stage A liver cancer accounts for $60 \%-65 \%$ of all cancers, which is significantly higher than the $10 \%-30 \%{ }^{[12]}$ diagnostic rate of early liver cancer worldwide, indicating thereby that the guidelines of stratified surveillance ares more conducive to the early diagnosis of liver cancer.

With the progress in basic and clinical epidemiological research on liver diseases, new evidences are emerging for the understanding of the high-risk PLC population. As safe and effective anti-viral drugs are widely used, more than $95 \%$ of the hepatitis $\mathrm{C}$ virus ( $\mathrm{HCV}$ ) is eradicated and $80 \%-90 \%$ of hepatitis B virus (HBV) is effectively inhibited, leading to changes in population numbers with liver cancer risk. Therefore, 
Table 1. Modifications of the GRADE system for strength of recommendations and quality of evidenceand

\begin{tabular}{|c|c|c|}
\hline GRADE system & Symbol & Definition \\
\hline $\begin{array}{l}\text { GRADE processed rating } \\
\text { evidence quality }\end{array}$ & & $\begin{array}{l}\text { In the GRADE approach, RCTs start as high-quality evidence and observational studies as } \\
\text { low-quality evidence supporting estimates of intervention effects. The quality of evidence for } \\
\text { each outcome falls into one of } 3 \text { categories from high to low }\end{array}$ \\
\hline High quality & A & It includes at least 1 RCT or literature meta-analysis/systematic review study \\
\hline Medium quality & B & $\begin{array}{l}\text { The estimated effect value is moderately reliable. Further study may change the reliability. } \\
\text { It includes non-randomized, controlled, well-designed cohort or case-control observational } \\
\text { studies }\end{array}$ \\
\hline Low quality & C & $\begin{array}{l}\text { Further study is highly likely to change the reliability of the estimated effect value. It includes } \\
\text { non-controlled clinical observational studies, case reports, or expert experience }\end{array}$ \\
\hline Grade of recommendation & & $\begin{array}{l}\text { The authors then consider the direction and strength of recommendation. The balance } \\
\text { between desirable and undesirable outcomes, and the application of patients' values and } \\
\text { preferences determine the direction of the recommendation. These factors, along with the } \\
\text { quality of the evidence, determine the strength of the recommendation }\end{array}$ \\
\hline Strong & 1 & $\begin{array}{l}\text { It is clearly demonstrated that the intervention brings benefits more than risks or the } \\
\text { opposite }\end{array}$ \\
\hline Weak & 2 & $\begin{array}{l}\text { Benefits and risks are uncertain, or demonstrated to be comparable, irrespective of the } \\
\text { evidence quality }\end{array}$ \\
\hline
\end{tabular}

RCTs: Randomized controlled trials.

in China, the Professional Committee for Prevention and Control of Hepatobiliary and Pancreatic Diseases of Chinese Preventive Medicine Association, the Professional Committee of Liver of Chinese Research Hospital Association, the Liver Society of Chinese Medical Association, the Prevention of Infection Related Cancer (PIRCA) Group, and the Specialist Committee of Cancer Prevention and Control of Chinese Preventive Medicine Association organized experts in the fields of liver, gastroenterology, hepatobiliary surgery, tumor intervention, infectious diseases and epidemiology to jointly develop this guideline. This guideline was developed in accordance with evidence-based medicine and the criteria of AGREE I $^{[13]}$, and a guidance group, writing group, discussion group (including communication experts), and evidence assessment group were established. The purpose of this guideline is to screen patients that are at risk of liver cancer with an exact etiology in hospital and community populations; provide guidance for scientific identification of high-risk population of liver cancer and stratified surveillance; assist physicians engaged in the specialties of liver disease or infectious diseases, digestion, hepatobiliary surgery, disease prevention and control; and provide a reference for the high-risk population screening and surveillance decisions. The guideline is not mandatory and does not possibly cover or address all issues of high-risk population screening and surveillance. Therefore, in considering an individual patient, the physician should develop a reasonable stratified screening and surveillance plan for liver cancer by following the principles of this guideline, based on a full understanding of the patient's disease characteristics and willingness, in combination with local medical resources and clinical practice.

The evidences and recommendations mentioned in this guideline are graded according to modifications of the GRADE system (assessment, development, and evaluation of recommended grading) [Table 1].

\section{TERMINOLOGY}

Screening and surveillance for liver cancer is a national public health program. It aims to achieve early diagnosis and treatment of liver cancer through community or hospital screenings to identify patients at risk of liver cancer and include them in a life-long surveillance program for liver cancer, thus reducing liver cancer related mortality and liver disease related overall mortality ${ }^{[14]}$.

\section{Screening}

In 1951, the National Association of Chronic Diseases officially introduced the concept of screening that is, distinguishing people who may be sick or defective, but appear to be healthy or asymptomatic, from those who are disease-free, by effective, simple, and economic examination methods. Screening 
not only detects patients at an early stage of chronic diseases, but also helps to identify the high-risk population of these diseases. It is classified into three types by population, i.e., opportunistic screening, targeted population screening, and extensive screening. Opportunistic screening is defined as that when an individual actively or voluntarily goes to a medical institution for examination of specific diseases; targeted population screening and extensive screening are defined as an organized examination of specific diseases provided for the targeted population in a community or work unit. We note that screening is not equal to a diagnostic examination.

\section{Surveillance}

Refers to the continuous, systematic, longitudinal, and regular examination of a high-risk population over time. In addition to the indicators of screening, imaging examinations such as contrast-enhanced ultrasonography, enhanced CT and/or MRI to detect BCLC 0- or A- type early liver cancer among patients with high risk is also known as enhanced screening ${ }^{[15]}$. Surveillance includes passive and active surveillance.

\section{Harms of screening and surveillance}

Screening and surveillance has the potential for physical, economical, and psychological harm to patients. In a single-center cohort study, 680 patients with liver cirrhosis received 3-year surveillance for HCC, and surveillance-related physical harms were reported in $27.5 \%$ of patients, including in $9.7 \%$ with multiple enhanced CT or MRI examinations and $0.4 \%$ with invasive examinations ${ }^{[16]}$. Although US and AFP do not cause direct physical harm to patients, the impending potential psychological harm associated with a possible positive diagnosis of PLC remains.

\section{PATHOLOGY AND PRECANCEROUS LESIONS OF LIVER CANCER}

According to the pathological classification, PLC was classified as HCC, ICC, and CHC. Due to the differences in pathogenesis and risk factor exposure, the pathological types of liver cancer vary slightly in different countries and regions. The incidence of ICC is gradually increasing among European and American populations ${ }^{[17]}$, while there is no exact report on the trend of HCC and ICC in China. The National Cancer Center of China reported pathological types of liver cancer in 2172 patients; HCC accounted for about $83.9 \%$ and ICC accounted for about $11.0 \%$ of all PLCs ${ }^{[18]}$. Due to the lack of specific serum and tissue markers, the diagnosis of early liver cancer and precancerous lesions or diseases remain debatable among clinicians and pathologists ${ }^{[19]}$. The occurrence and development of liver cancer is a damaging process involving multiple gene mutations and signaling pathways, generally sequencing from chronic hepatitis, liver cirrhosis, cirrhotic hyperplastic nodules, low-grade dysplastic nodules (LGDN), high-grade dysplastic nodules (HGDN) to pre-early liver cancer, early liver cancer, and finally, advanced liver cancer; thereby following a multi-step process from molecular to clinical evolution ${ }^{[20]}$. A study by Japanese scholars shows that the 1-, 3-, and 5-year cumulative incidence of progression from cirrhotic hyperplastic nodules to liver cancer is $3.3 \%, 9.7 \%$, and $12.4 \%$, respectively. The 1-, 3-, and 5-year cumulative incidence of progression from LGDN (called precancerous lesions) to liver cancer is $2.6 \%, 30.2 \%$, and $36.6 \%$, respectively, and the 1-, 3-, and 5-year cumulative incidence of progression from HGDN (called precancerous lesions) to liver cancer is $46.2 \%, 61.5 \%$, and $80.8 \%$, respectively ${ }^{[21]}$. In addition, liver cancer also shows a trend of doubling growth of tumor size (cut-off value is usually considered as $2 \mathrm{~cm}$ ) over $t_{i m e} e^{[22]}$. Therefore, there is sufficient opportunity in clinical practice to identify liver cancer during surveillance of precancerous lesions or diseases with tumor $<2 \mathrm{~cm}$, and thereby achieve the goal of early diagnosis and treatment of liver cancer. Therefore, it is of great significance to strengthen the understanding of precancerous diseases and lesions for accurately identifying the high-risk population and early diagnosis of liver cancer.

\section{EPIDEMIOLOGY AND DISEASE BURDEN}

85\%-95\% of PLC occurs secondary to cirrhosis pathologically diagnosed by liver biopsy as a major risk factorand. Radiological diagnostic criteria, instead of pathological diagnosis, should be adopted for 
the diagnosis of PLC in patients with existing cirrhosis. Therefore, statistical methods are usually used worldwide, including China, to estimate the incidence and causes of PLC. These include calculations by the International Classification of Diseases 10 (ICD-10) and exact pathology data, or multivariate estimation methods, which may produce a result somewhat deviating from the actual incidence ${ }^{[23]}$.

\section{Incidence and mortality}

Based on the GLOBOCAN data in 2018, there were about 792,031 new cases of PLC worldwide as per ICD-10. The ASR incidence was 8.9/100,000 wordwide, 13.4/100,000 in males, and 4.7/100,000 in females; ranking PLC as the sixth most common malignant tumors worldwide, fifth most common in men and the ninth most common in women, respectively ${ }^{[24]}$. Mongolia has the highest incidence of PLC in the world, with an ASR incidence of 90.0/100,000. It also suffers the highest mortality of PLC in the world, with about 727,661 PLC deaths and an ASR mortality of 8.1/100,000, ranking fourth in the spectrum of malignant tumor deaths, the male mortality and female mortality ranking the second and sixth in the spectrum of malignant tumor deaths, respectively ${ }^{[24]}$. The incidence and mortality of PLC are low in European and American areas, but have gradually increased in the past 10-year; the incidence of PLC is high in Asian population, but both incidence and mortality are decreasing year-by-year ${ }^{[24]}$.

In 2018, GLOBOCAN reported an ASR incidence of 17.7/100,000 for PLCand the ASR mortality as $16.4 / 100,000$ in the Chinese population, both of which ranked ninth in the world. In 2014, there were about 364,800 new cases of PLC in China, ranking fourth in the incidence of all tumors and 318,800 deaths, ranking second in all tumor deaths. The total incidents and death cases of PLC accounted for about half of the world's cases each year, with significant urban-rural and regional differences; the population-adjusted incidence in rural area was $20.07 / 100,000$ and the mortality was $17.52 / 100,000$, higher than the incidence $(16.13 / 100,000)$ and mortality $(13.64 / 100,000)$ in urban areas ${ }^{[23]}$. At present, the incidence $(20.85 / 100,000)$ and mortality $(16.98 / 100,000)$ in western China are higher than those in central $(18.23 / 100,000$ and $15.97 / 100,000$, respectively) and eastern $\left(15.31 / 100,000\right.$ and $13.52 / 100,000$, respectively) ${ }^{[23]}$. Due to improvements in living environment, hepatitis B vaccination, and effective antiviral therapy for chronic HBV infection in China ${ }^{[25]}$, the standardized incidence of PLC in China decreased by $2.3 \%$ per year during 2000-2014, including 3.9\% per year in the age group $<40$ years ${ }^{[23]}$. At the same time, the average age of onset of liver cancer was delayed from 59.67 and 56.53 years in 2000 to 62.66 and 61.20 years in 2014, in urban and rural males, respectively, and from 65.50 and 60.60 years to 69.87 and 66.07 years in urban and rural females respectively ${ }^{[26]}$.

\section{Disease burden}

Globally, PLC ranks second in malignant tumor deaths and disability-adjusted life-years (DALYs) ${ }^{[24]}$. In 2017, among all chronic diseases in China, PLC became the fifth leading cause of years of life lost (YLLs), and the seventh leading cause of DALY ${ }^{[27]}$. During 1990-2017, both DALYs and YLLs were increasing, with DALYs increasing by $43.5 \%$ (95\%UI: $31.2-60.3)$ and YLLs by $21.2 \%$ (95\%UI: $10.8-35.2)$ in all age groups ${ }^{[27]}$. Aging is a major cause of increasing disease burden of PLC ${ }^{[24,26]}$.

Recommendation 1: Screening and surveillance for liver cancer should be included in national public health programs, aiming to reduce liver cancer related mortality and liver diseases related overall mortality (C1).

Recommendation 2: The age of onset of liver cancer in China is gradually increasing and the age-adjusted incidence is also decreasing year by year, but the resulting disease burden is still on the rise (A1).

\section{ETIOLOGY OF LIVER CANCER}

More than $90 \%$ of liver cancers have a clear etiology and include cirrhosis, HBV infection, HCV infection, alcohol, nonalcoholic fatty liver disease (NAFLD, now renamed metabolism-related fatty liver disease by a 
consensus of international experts ${ }^{[28]}$. However, we use the term NAFLD in this guideline), diabetes, longterm exposure to carcinogens such as aflatoxin and aristolochic acid, and a family history of liver cancer that known to significantly increase the risk of onset in virus-infected populations.

\section{Liver cirrhosis}

Liver cirrhosis from various causes is the most hazardous link in the development of HCC, and $85 \%-95 \%$ of HCC cases have pre-existing cirrhosis ${ }^{[29]}$. The annual incidence of liver cancer in patients with HBV-related cirrhosis ranges from $3 \%-6 \%$, which is the leading cause of HCC in $\mathrm{China}^{[30]}$, and the annual incidence of liver cancer developed from HCV-related cirrhosis ranges from $2 \%-4 \%{ }^{[31]}$. The risk of developing liver cancer is significantly reduced in patients with HBV and/or HCV-related cirrhosis who achieve sustained virological response (SVR) after antiviral therapy. A cohort study of 2,079 patients with cirrhosis of multiple etiologies and followed-up for 10-year showed that the 10-year cumulative incidence of HCC was $16.3 \%$ and $4.6 \%$ in patients with NAFLD and autoimmune cirrhosis, respectively ${ }^{[32]}$. Compared with those with HBV and/or HCV-related cirrhosis, patients with alcoholic cirrhosis have a 2- to 3-fold lower absolute risk of developing $\mathrm{HCC}^{[33]}$. The risk of progression from cirrhosis to HCC is influenced by factors such as age, gender, etiology, family history of liver cancer, and diabetes. Among patients with cirrhosis, old age, male, combined low platelet count $\left(<100 \times 10^{9} / \mathrm{L}\right)$, and esophageal varices contribute to a higher risk of HCC. It has been shown that the incidence of HCC is correlated with portal hypertension or the degree of liver stiffness measured by liver stiffness measurement (LSM) using transient elastography ${ }^{[34]}$. Cirrhosis induced by chronic biliary diseases, hemochromatosis, hepatic porphyria, and $\alpha 1$-antitrypsin deficiency may increase the risk of HCC, but this accounts for only a small proportion worldwide.

\section{Chronic HBV infection}

HBV infection is the leading cause of HCC worldwide, especially in East Asian and African countries ${ }^{[35]}$. The results of etiological analysis performed by the National Cancer Center of China in 1,823 patients with HCC showed that $86.0 \%$ of these patients had HBV simplex infectiond and $6.7 \%$ had mixed HBV and HCV infections ${ }^{[18]}$. Another study has shown that occult HBV infected patients with negative hepatitis B surface antigen (HBsAg) have a higher risk of HCC than non-HBV infected patients, with a post-adjustment relative risk (RR) of 2.86 (95\%CI: $1.59-4.13)^{[36]}$. A higher risk of HCC was observed in HBV-infected patients with a family history of liver cancer. A study of 22,472 residents followed-up for $16.12( \pm 2.89)$ years showed that in HBsAg-negative population, the cumulative incidence of HCC was $0.62 \%$ in people without a family history of liver cancer and $0.65 \%$ in those with a family history of liver cancer; however, in HBsAg-positive people, the cumulative incidence of HCC was $7.5 \%$ in people without a family history of liver cancer and $15.8 \%$ in those with a family history of liver cancer $^{[37]}$.

Hepatitis B e antigen (HBeAg) status, peripheral blood HBV DNA load, HBV genotype, and relevant genetic mutations are associated with HCC risk ${ }^{[38-46]}$. In Qidong, a high incidence area of liver cancer in China, the RR of HCC in HBsAg positive patients who were either HBeAg negative or HBeAg positive was 13.25 (95\%CI: 6.67-26.33) and 28.05 (95\%CI: 13.87-56.73) ${ }^{[38]}$, respectively, compared with HBsAg and HBeAg negative patients. It is notable that the cumulative incidence of HCC is not linearly positively correlated with HBV DNA level. The hazard ratio (HR) of HCC is highest at HBV DNA loads of $10^{4}-10^{6}$ copies $/ \mathrm{ml}$, but decreases when HBV DNA load is greater than $10^{6}$ copies $/ \mathrm{ml}^{[39]}$. In HBeAg negative patients who had HBV-DNA levels of 2,000-20,000 IU/mL andhepatitis B core-related antigen (HBcrAg) $\geq 10 \mathrm{KU} / \mathrm{mL}$, the risk of HCC significantly increased ${ }^{[40]}$. The risk of HCC increased by 13.7 -fold (95\%CI: 4.8-39.7) in HBeAg negative patients with HBV-DNA $<2,000 \mathrm{IU} / \mathrm{mL}$, but $\mathrm{HBsAg} \geq 1000 \mathrm{IU} / \mathrm{mL}$, compared with those who had $\mathrm{HBsAg}<1000 \mathrm{IU} / \mathrm{mL}^{[41]}$. Therefore, in an era of antiviral therapy, HBsAg and $\mathrm{HBcrAg}$ quantification is complementary to HBV DNA as a marker to predict progression to HCC in HBV infected patients ${ }^{[40,42]}$. However, after 50 -year of age, HBV-infected patients in whom HBsAg has disappeared, continue to face a higher risk of HCC. The mutations in HBV genotype and gene can significantly increase 
the risk of HCC, including mutations in the pre-S region of HBV (OR: 3.77, 95\%CI: 2.57-5.52), C1653T mutation in the II region of enhancer (OR: 2.76, 95\%CI: 2.09-3.64), T1753V mutation (OR: $2.35,95 \% \mathrm{CI}$ : 1.63-3.40), and A1762T/G1764A double mutation in the core promoter region (OR: 3.79, 95\%CI: 2.71-5.29), all of which are independent predictors of HCC risk $^{[43]}$. Moreover, these mutations show an accumulation tendency during the development of $\mathrm{HCC}^{[44-47]}$.

Moreover, the use of nucleos(t)ide analogues (NAs), first-line antiviral drugs - entecavir (ETV), tenofovir disoproxil fumarate (TDF), and tenofovir alafenamide fumarate (TAF) significantly reduced the risk of progression to cirrhosis and HCC in HBV patients ${ }^{[47-50]}$. Interferon-alpha (IFN- $\alpha$ ) is another kind of antiviral agent that can also reduce the risk of progression to liver cancer in patients with chronic $\mathrm{HBV}^{[51]}$. However, the risk of progression of chronic HBV infection to liver cancer cannot be eliminated regardless of NAs or IFN- $\alpha$ treatment.

\section{Other etiologies}

\section{Chronic HCV infection}

HCC developed from HCV infection is attributable to cirrhosis caused by HCV. In North America, 59\% (95\%CI: $3 \%-79 \%$ ) of HCC is attributable to HCV infection; in Northern Europe, $28 \%$ (95\%CI: $10 \%-55 \%$ ); in the rest of Europe, $40 \%$ (95\%CI: 32\%-49\%); and in North Central Africa, $79 \%$ (95\%CI: $69 \%-86 \%)^{[52]}$. In China, HCV simplex infection accounts for only $1.7 \%-2.5 \%$ of all causes of HCC, while HCV and HBV combined infection accounts for $6.7 \%^{[18,53]}$. Family history of liver cancer can also increase the risk of HCC in HCV-infected patients. A meta-analysis of the western population showed that the OR of HCC risk was 2.94 (95\%CI: 0.94-9.21) in people with a family history of liver cancer but negative HBsAg and anti-HCV antibodies; whereas in people with positive viral infection, the OR of HCC risk was 38.19 (95\%CI: 21.9766.39) in people without a family history of liver cancer and 72.48 (95\%CI: 21.92-239.73) in people with a family history ${ }^{[54]}$. It has been shown that pegylated interferon (PegIFN) or direct-acting antiviral drugs (DAA) can reduce the risk of HCV-related cirrhosis and HCC ${ }^{[55]}$. Among HCV-infected individuals, high risk factors for HCC include male, older age, combined human immunodeficiency virus or HBV infection, diabetes, and chronic alcohol consumption ${ }^{[9,56-59]}$.

\section{Alcoholic liver disease, NAFLD, with or without diabetes}

Alcoholic liver disease is the leading cause of HCC in European and American countries, second only to HCV or HBV infection. A French multicenter cohort study of 652 patients with alcoholic cirrhosis showed that the annual incidence of HCC was $2.9 \%$, and alcohol abuse (ethanol $\geq 80 \mathrm{ml} / \mathrm{d}$ ) significantly increased the risk of progression of cirrhosis to $\mathrm{HCC}^{[56]}$. A case-control study showed that the OR for the risk of progression to HCC from alcoholic cirrhosis alone, HCV cirrhosis, or HBV cirrhosis was 4.5 (95\%CI: 1.414.8), 15.3 (95\%CI: 4.3-54.4), and 12.6 (95\%CI: 2.5-63.1), respectively. The OR was as high as 53.9 (95\%CI: 7.0-415.7) in patients with two or more of these factors, i.e., alcoholic liver disease accompanied with chronic HCV or chronic HBV infection ${ }^{[57]}$. There is an interaction between smoking, obesity, diabetes, and alcohol to enhance the risk of $\mathrm{HCC}^{[56]}$. A meta-analysis showed that the risk of HCC can decrease by $6 \%$ $7 \%$ per year after alcohol withdrawal, but it takes 23 -year (95\%CI: 14-70) to decrease to the risk level of non-drinkers ${ }^{[59]}$.

NAFLD is currently the most common liver disease worldwide and is emerging as an important cause of HCC $^{[60,61]}$. A study involving $~ 130,000$ patients with NAFLD or nonalcoholic steatohepatitis (NASH) in four European cohorts showed that patients with NAFLD or NASH had a 3.51-fold (95\%CI: 1.72-7.16) higher risk of liver cancer compared with the general population, and the proportion of patients with NAFLD was as high as $26.3 \%$ in HCC patients without a cirrhosis background ${ }^{[62]}$. In a prospective cohort study in community population conducted in China (US-diagnosed NAFLD), only NASH patients with abnormal liver function, i.e., alanine aminotransferase (ALT) $\geq 80 \mathrm{U} / \mathrm{L}$, had a 4.41 -fold (95\%CI: 1.40-13.96) higher 
risk of HCC compared with controls ${ }^{[63]}$. Since some NAFLD cases are also accompanied with alcoholic liver disease, the exact risk of NAFLD for HCC remains to be explored. NAFLD patients with diabetes face a 1.8- to 2.5-fold higher risk of developing HCC compared with normal people, which is an independent factor for the pathogenesis and prognosis of HCC in all races ${ }^{[64,65]}$.

\section{Exposure to carcinogens}

Exposure to aflatoxin B1 (AFB1) is an important cause of the high incidence of HCC in sub-Saharan Africa, Southeast Asia, and some rural humid regions of China. AFB1 was classified as a Class I carcinogen by the International Agency for Research on Cancer (IARC) in 1987. The direct carcinogenic effect of AFB1 is controversial, but studies have demonstrated a strong interaction between AFB1 and HBV infection ${ }^{[6,67]}$. A case-control study showed that in the HBsAg negative population, the risk of HCC caused by an increased exposure to AFB1 alone was 1.9-fold higher than that in the non-exposed population, and the risk of HCC could be increased to 60.1-fold when chronic HBV infection was superimposed ${ }^{[66]}$. With the implementation of water and grain improvement measures by the Chinese government, AFB1 exposure have decreased dramatically in the population of high incidence areas of liver cancer in China, and its contribution to the etiology of HCC in China is also gradually weakening ${ }^{[67]}$.

Aristolochic acid (AA), which is present in plants such as Aristolochia and Asarum, is an ingredient of some Chinese herbal prescriptions. In 2012, AA was classified as a Class I carcinogen by IARC. Analysis of genomic features of HCC suggests that the proportion of hepatocellular carcinoma with AA characteristic mutations in Asian population is much higher than that in European and American populations, especially as high as $78 \%$ in Taiwan, China ${ }^{[68]}$. In patients with hepatitis B, there is a dose-response relationship between AA exposure and risk of $\mathrm{HCC}^{[69]}$. However, these reports need to be independently evaluated in prospective cohort studies.

\section{Changes in the etiology of liver cancer}

Due to neonatal hepatitis B vaccination programs and effective control of aflatoxin exposure, the incidence of HBV-induced HCC in young adults has decreased significantly ${ }^{[25,67,70]}$. Screening for HBV and HCV infection, and a timely and effective antiviral therapy have led to a gradual decrease in the proportion of $\mathrm{HBV}$ and $\mathrm{HCV}$-related $\mathrm{HCC}^{[71]}$, while the increasing proportion of non-infectious liver diseases caused by alcohol and NAFLD lead to a rising incidence of HCC. The changes in etiologies of chronic liver diseases have caused a gradual change in the etiologies of HCC worldwide.

Recommendation 3: Chronic HBV infection is the main cause of HCC (A1). The incidence of liver cancer in patients with alcohol- and metabolism-related diseases is increasing year by year (B1). Aflatoxin B1 increases the risk of liver cancer in patients with HBV infection, alcoholic liver disease (A1).

Recommendation 4: Cirrhosis of various causes is a hazardous link in the development of liver cancer, and HBV-related cirrhosis is the primary cause of HCC in China (A1).

\section{IDENTIFICATION AND STRATIFICATION OF HIGH-RISK POPULATION OF LIVER CANCER}

Patients with cirrhosis and chronic HBV or HCV infection who have not received antiviral therapy or acquired SVR are the major high-risk populations of HCC in China.

\section{Risk stratification of HCC in patients with cirrhosis}

Cirrhotic patients are recognized as high-risk population of HCC as per worldwide guidelines. However, the risk of progression of cirrhosis to HCC varies with different etiologies. Therefore, the Toronto HCC risk index (THRI) included four variables: age, gender, etiologies of cirrhosis (o for autoimmune liver disease, 0 for hepatitis C with SVR, 36 for other liver diseases, 54 for steatohepatitis, and 97 each for untreated chronic hepatitis $\mathrm{C}$ or chronic hepatitis $\mathrm{B}$ ), and platelet count to construct model with a total of 
Table 2. Toronto HCC risk index model parameters and assignment

\begin{tabular}{ll}
\hline Variable & Score (points) \\
\hline Gender & 0 \\
Female & 80 \\
Male & \\
Age (year) & 0 \\
$<45$ & 50 \\
$45-60$ & 100 \\
$>60$ & \\
Etiology of cirrhosis & 0 \\
Autoimmune liver disease & 0 \\
Hepatitis C with SVR & 36 \\
Other causes of liver disease & 54 \\
Steatohepatitis & 97 \\
Hepatitis C & 97 \\
Hepatitis B & \\
Platelet count $(/ \mathrm{L})$ & 0 \\
$>200 \times 10^{9}$ & 20 \\
$(140-200) \times 10^{9}$ & 70 \\
$(80-139) \times 10^{9}$ & 89 \\
$<80 \times 10^{9}$ & 366 \\
Total score &
\end{tabular}

SVR: Sustained virologic response.

366 points [Table 2]. The 5-year cumulative incidence of HCC was $1.2 \%, 4.4 \%$, and $15.4 \%$, and the 10-year cumulative incidence of HCC was $3 \%, 10 \%$, and $32 \%$ in the low-risk (0-120), intermediate-risk (120-240), and high-risk (>240) groups, respectively ${ }^{[32]}$. The THRI model has also been well validated in China, with a 5 -year cumulative incidence of HCC being $0 \%, 13 \%$, and $34 \%$ in cirrhotic patients classified in the low, intermediate-, and high-risk groups, respectively, and the area under the receiver operating characteristic curve (AUROC) of $0.707^{[72]}$. The significance of the THRI model is that even in cirrhotic patients with high risk of HCC, further risk stratification is necessary, that is, it can distinguish the population with extremely high risk of HCC. Patients with the following characteristics in addition to cirrhosis are at higher risk of HCC: (1) US-revealed liver nodules (1-2 cm) or LGDN and HGDN in pathology. Indeed, the annual incidence of progression of LGDN and HGDN to liver cancer is about $10 \%$ and $20 \%$, respectively ${ }^{[21]}$; (2) Patients with cirrhosis and diabetes, who have a 2- to 3 -fold higher risk of HCC compared with those without diabetes ${ }^{[73]}$; and (3) Patients with cirrhotic nodules $(\leq 1 \mathrm{~cm})$ who have not received antiviral drugs or still have persistent low viral load of HBV DNA after treatment ${ }^{[74]}$.

In recent years, LSM as a noninvasive means of cirrhosis examination is gradually demonstrating its value in the risk stratification of HCC. A Korean prospective study showed that among HBV-infected patients with LSM of 8.1-13 kPa, 13.1-18 kPa, 18.1-23 kPa, and > $23 \mathrm{kPa}$, the HR of developing HCC was 3.07, 4.68, 5.55, and 6.60, respectively ${ }^{[35]}$. In HCV-infected patients in Taiwan, the 5-year cumulative incidence of HCC was only $0.9 \%$ when LSM was $<12.0 \mathrm{kPa}$, while when the LSM was $12.0-24.0 \mathrm{kPa}$ and $>24.0 \mathrm{kPa}$, the 5-year cumulative incidence of HCC increased to $9.5 \%$ and $45.1 \%$, respectively ${ }^{[75]}$. Therefore, in patients with chronic liver disease due to all causes, attention should be paid to the early diagnosis and screening of cirrhosis.

\section{Risk stratification of HCC in HBV-infected patients}

Although HBV infection is the main cause of HCC, in the absence of cirrhosis, only $6.2 \%$ to $9.5 \%$ of HBVinfected individuals progress to HCC in their lifetime. HBV-infected patients in the immune tolerant phase (ALT and AST continue to be normal, HBeAg positive, HBV DNA $>10^{7} \mathrm{IU} / \mathrm{ml}$; no significant inflammation or fibrosis proven by biopsy), have a 10 -year cumulative incidence of liver cancer as low as $1.7 \%{ }^{[76]}$. Scholars 
Table 3. REACH-B model parameters and assignment

\begin{tabular}{lc}
\hline Variable & Score (points) \\
\hline Gender & 0 \\
Female & 2 \\
Male & \\
Age (year) & 0 \\
$30-34$ & 1 \\
$35-39$ & 2 \\
$40-44$ & 3 \\
$45-49$ & 4 \\
$50-54$ & 5 \\
$55-59$ & 6 \\
$\geq 60$ & \\
HBeAg status & 2 \\
Positive & 0 \\
Negative & \\
HBV DNA (copy/ml) & \\
$<10^{4}$ & 0 \\
$10^{4}-10^{5}$ & 3 \\
$10^{5}-10^{6}$ & 5 \\
$>10^{6}$ & 4 \\
ALT (U/L) & \\
$<15$ & 0 \\
$15-44$ & 0 \\
$\geq 45$ & 17 \\
Total score & \\
\hline
\end{tabular}

HBeAg: Hepatitis B E antigen; HBV DNA: deoxyribonucleic acid of hepatitis b virus; ALT: alanine aminotransferase.

from Taiwan, China constructed a HCC predictive scoring system, REACH-B ${ }^{[39]}$, with a total of 17 points that include five parameters: gender, age, ALT, HBeAg status, and HBV DNA level [Table 3]. The purpose of REACH-B was tostratify the risk of HCC development in HBV-infected patients receiving no antiviral therapy, and the scores of 0-5, 6-11, and 12-17 corresponded to low risk, intermediate risk, and high risk, respectively. The incidence of HCC within 3-, 5-, and 10-year was $0.0 \%$ and $23.6 \%, 0.0 \%$ and $47.4 \%$, and $0.0 \%$ and $81.6 \%$ in patients with the lowest (0) and highest scores (17), respectively. In Qidong, a high incidence area of liver cancer in China, an AGED model (12 points in total) ${ }^{[38]}$ was constructed using four parameters: gender, age, HBeAg status, and HBV DNA level. The study found that therisk of developing HCC within 20-year was 20.3-fold higher in the high-risk group ( $\geq 10$ points) than in the low-risk group ( $\leq 4$ points). In particular, the risk of HCC in the low-risk group was generally consistent with that in the general population without HBV infection, suggesting that it is not necessary for HBV-infected patients in the low-risk group to receive the same HCC surveillance regimen as the high-risk group.

A PAGE-B model was constructed based on the results of 5-year antiviral therapy with oral ETV or TDF in 1,815 patients with chronic hepatitis B from nine prospective cohorts in Europe. The model included age, gender, and platelet count as the main parameters, with a total score of 25 points [Table 4] for assessing the risk of HCC in HBV-infected patients after antiviral therapy ${ }^{[77]}$. PAGE-B classifies the patients into low-risk (0-9 points), intermediate-risk (10-17 points), and high-risk (18-25 points) groups. In both the discovery and validation cohorts, the 5-year cumulative incidence of HCC was 0 in low-risk patients, 3\%$4 \%$ in intermediate-risk patients, and $16 \%-17 \%$ in high-risk patients. The Korean team validated that the PAGE-B model was also effective in Asians, with an AUC of 0.77 for a 5-year HCC predictive power ${ }^{[78]}$. Recently, the PAGE-B study team constructed a SAGE-B model for reassessing the risk of HCC after 5-year of antiviral therapy ${ }^{[79]}$, including 2 parameters: age and LSM after 5 -year of antiviral therapy, totaling to 15 points. SAGE-B classifies the patients into low-risk ( $\leq 5$ points), intermediate-risk (6-10 points), and highrisk ( $\geq 11$ points), in which the 12 -year cumulative incidence of HCC was $0 \%, 4 \%$, and $13.8 \%$, respectively. 


\begin{tabular}{lc} 
Table 4. PAGE-B model parameters and assignment \\
\hline Variable & Score (points) \\
\hline Gender & \\
Female & 0 \\
Male & 6 \\
Age (year) & \\
$16-29$ & 0 \\
$30-39$ & 2 \\
$40-49$ & 4 \\
$50-59$ & 6 \\
$60-69$ & 8 \\
$\geq 70$ & 10 \\
Platelet count $\left(/ \mathrm{mm}^{3}\right)$ & \\
$\geq 200000$ & 0 \\
$100000-199999$ & 6 \\
$<100000$ & 9 \\
Total score & 25 \\
\hline
\end{tabular}

In conclusion, even in patients with chronic HBV infection treated with antiviral therapy, the 5-year cumulative incidence of HCC was as high as $5.7 \%-8.4 \%$, especially in high-risk patients. But it is worth pointing out that the 5 -year ${ }^{[78]}$ and 12 -year ${ }^{[79]}$ negative HCC prediction rates were $97.5 \%-100 \%$ in low-risk patients, suggesting that these patients can be spared from routine liver cancer surveillance twice a year.

In conclusion, the risk factors for an increased risk of HCC in HBV-infected individuals are being male, age 40-year or older, family history of liver cancer in a first-degree relative, and diabetes. In addition, the failure of antiviral therapy or drug resistance, such as $\mathrm{rtA} 181 \mathrm{~T}$, rtL80I, rtN236 $\mathrm{T}$ mutations, or the presence of lowlevel viremia (LLV) can also increase the risk of $\mathrm{HCC}^{[80]}$.

\section{Risk stratification of HCC in other populations}

Risk stratification of HCC in HCV-infected patients

Serum HCV RNA, ALT, and HCV genotype1b are independent predictors of HCC in treatment-naive HCV infected patients ${ }^{[31,81]}$. In HCV-infected patients receiving treatment, age and degree of liver fibrosis are major factors in the development of HCC. In patients with chronic HCV infection who achieved SVR after PegIFN or DAA therapy, especially those with cirrhosis, the risk of HCC cannot be completely eliminated, though significantly reduced ${ }^{[5,82]}$. Therefore, there is no reference index for stopping surveillance in patients included in the surveillance of HCC at an early stage, and the surveillance should be continued as originally planned.

\section{Risk stratification of HCC in patients with NAFLD and alcoholic liver disease}

Less importance is given to the screening and surveillance of HCC in patients with NAFLD, especially at the non-cirrhotic stage than in HBV- or HCV-infected patients. This is mainly because NAFLD has a high prevalence in the general population ${ }^{[83]}$, and the risk of HCC in NAFLD and NASH patients is lower than in HBV or HCV infected patients. It is not cost-effective to screen all NAFLD patients. Therefore, risk stratification for HCC is particularly important in NAFLD patients. A multicenter prospective cohort study conducted in 136,703 NAFLD/NASH patients from 4 countries in Europe showed that Fib-4 index (age $\times$ AST/platelet count $\times \sqrt{A L T}$ ) that predicts liver fibrosis might be used to stratify the risk of HCC in NAFLD/NASH patients. Compared to patients with Fib-4 $<1.3$ (no significant fibrosis), those with Fib-4 of 1.30-2.67 (moderate fibrosis) and Fib-4 > 2.67 (severe fibrosis/cirrhosis) have a HCC HR of 3.74 and 25.2, respectively ${ }^{[60]}$. Therefore, attention should be paid to screening liver fibrosis and cirrhosis in these patients, especially when stratification evidence such as detecting fibrosis via non-invasive LSM for the progression to HCC from alcoholic liver disease is lacking. 
Family history of liver cancer

A family history of liver cancer in first-degree relatives is a factor that significantly increases the risk of $\mathrm{HCC}$ in people infected with $\mathrm{HBV}$ or $\mathrm{HCV}^{[37,54,84]}$. Scholars from Taiwan, China evaluated 22,472 residents over 362,268 person-years of follow-up, in which 374 people developed HCC. Among HBsAg-positive patients, a family history of liver cancer increased the risk of HCC by 2.54 times ${ }^{[37]}$. A comparative study including 2,011 cases of liver cancer and 7,933 cases of non-liver cancer in Jiangsu Province of China showed that the OR of increased risk of HCC was 2.76 (95\%CI: 1.88-4.05) in HBV-negative patients with a family history of liver cancer, but up to 41.34 (95\%CI: 23.69-72.12) in HBV-positive patients with a family history of liver cancer in first-degree relatives ${ }^{[84]}$. Therefore, special attention should be paid to the family history of liver cancer in first-degree relatives since it leads to a significantly increased risk of HCC at all stages in HBV-infected ${ }^{[37,84]}$ or HCV-infected ${ }^{[54]}$ patients.

Although HBV or HCV infection is the main cause of HCC, the proportion of non-viral HCC is increasing year by year. Recently, Chinese scholars have constructed a 100-point aMAP model composed of five parameters including age, gender, platelets, albumin, and bilirubin using the data of 11 prospective cohorts $(n=17,374)$ worldwide for the prediction of HCC risk in patients with liver diseases, regardless of etiology. In patients assigned to the low-risk group ( $<50$ points), the 3 - and 5 -year cumulative incidence of HCC were as low as $0 \%$ and $0.8 \%$. Since the annual incidence of HCC in these patients was $<0.2 \%$, the authors suggested that the surveillance held every 6 months may not be required. However, the 3-year cumulative incidence of HCC was $1.5 \%$ and $4.8 \%$, and the 5 -year cumulative incidence of HCC was $8.1 \%$ and $19.9 \%$ in patients classified into the intermediate-risk (50-60 points) and high-risk (60-100 points) groups, respectively. However, this study found that low-risk patients accounted for $45 \%$, while the highrisk patients accounted for only $17.9 \%$ of all non-viral HCC $^{[85]}$. Therefore, the role of the aMAP model in identifying high-risk patients for development of HCC in patients with non-viral liver disease needs further evaluation.

In conclusion, a stratified management of high-risk population of liver cancer may be the most important strategy to improve its early diagnosis and cost-effectiveness. See Table 5 for identification and stratification of high-risk population of liver cancer.

\section{SCREENING AND SURVEILLANCE TOOLS}

\section{Ultrasonography}

US is recommended as a screening method for liver cancer by guidelines in many countries due to its ease of operation, flexibility, non-invasion, and low price ${ }^{[86]}$. US can sensitively detect space-occupying lesions in the liver and accurately distinguish cystic lesions from solid lesions. US can help identify liver tumors more than $2 \mathrm{~cm}$ in diameter for their benign or malignant nature according to the blood supply and other characteristics of the lesion. Overall, the sensitivity of US for the diagnosis of liver tumors $<2$ $\mathrm{cm}, 2-3 \mathrm{~cm}, 4-5 \mathrm{~cm}$, and $>5 \mathrm{~cm}$ in diameter was $39 \%-65 \%, 76 \%, 84 \%$, and $90 \%$, respectively ${ }^{[87]}$. Ultrasound Doppler not only detects blood supply within the lesion, but also identifies the relationship between the lesion and the vessel. Contrast-enhanced US visualizes hemodynamic changes within the liver mass, helps in the differential diagnosis of the nature of various hepatic space-occupying lesions, and improves the sensitivity and specificity of US in the diagnosis of liver cancer ${ }^{[88,89]}$. However, US is easily affected by the experience, operation, and meticulousness of the operator and by the obesity of patients. Regardless of its low sensitivity in the early diagnosis of liver cancer, US is still the most important tool for liver cancer screening and surveillance.

\section{CT and MRI}

Multi-slice spiral CT and MRI are the most important tools for the diagnosis of liver cancer and clinical staging, but the equipments are expensive and not practical to use in primary hospitals. Significantly 


\begin{tabular}{|c|c|c|c|}
\hline Recommendation & $\begin{array}{c}\text { Grade of risk for liver cancer, } \\
\text { Estimated annual incidence of } \\
\text { liver cancer (\%) }\end{array}$ & Identification features & $\begin{array}{l}\text { Risk stratification model } \\
\text { of liver cancer }\end{array}$ \\
\hline Recommendation 5 & Low-risk, $(<1)$ & $\begin{array}{l}\text { (1) HBV infection at immune tolerant phase (A1); } \\
\text { (2) HBV or HCV-related chronic hepatitis with } \\
\text { SVR to antiviral therapy (A1); (3) non-viral liver } \\
\text { disease with normal ALT, platelet counts (B1) }\end{array}$ & $\begin{array}{l}\text { Positive } \mathrm{HBsAg} \text {, REACH-B } \\
\text { score } \leq 5 \text { (no antiviral } \\
\text { therapy), or PAGE-B score } \\
\leq 9 \text { (B2) }\end{array}$ \\
\hline Recommendation 6 & Intermediate-risk, (1-3) & $\begin{array}{l}\text { (1) < 40-year of age: HBV- or HCV-related } \\
\text { chronic hepatitis without antiviral therapy or } \\
\text { with LLV after antiviral therapy (B1); HBV-or } \\
\text { HCV-related cirrhosis achieving SVR to antiviral } \\
\text { therapy (B1); ( } 2 \text { ) non-viral cirrhosis with normal } \\
\text { ALT or chronic non-viral hepatitis with abnormal } \\
\text { ALT (C2) }\end{array}$ & $\begin{array}{l}\text { (1) HBsAg positive, } \\
\text { REACH-B 6-11 (no antiviral } \\
\text { therapy) or PAGE-B } 10-17 \\
\text { (B2); (2) THRI score } \leq 240 \\
\text { in patients with cirrhosis } \\
\text { (B2) }\end{array}$ \\
\hline Recommendation 7 & High-risk, $(>3, \leq 6)$ & $\begin{array}{l}\text { (1) HBV- or HCV-related cirrhosis without } \\
\text { antiviral therapy or with LLV after antiviral } \\
\text { therapy (A1); ( } 2 \text { ) Non-viral cirrhosis patients } \\
\text { with diabetes or family history of liver cancer in } \\
\text { first-degree relative (B1); (3) Male, > } 40 \text {-year of } \\
\text { age; female, > 50-year of age; HBV/HCV-related } \\
\text { chronic hepatitis without antiviral therapy (B1) }\end{array}$ & $\begin{array}{l}\text { (1) } \mathrm{HBsAg} \text { positive, } \\
\text { REACH-B } \geq 12 \text { (no antiviral } \\
\text { therapy) or PAGE-B } \geq 18 \\
\text { (B2); ( } 2 \text { ) THRI score } \geq 240 \\
\text { in patients with cirrhosis } \\
\text { (B2) }\end{array}$ \\
\hline Recommendation 8 & Extremely high-risk, $(>6)$ & $\begin{array}{l}\text { (1) Liver nodules }(1-2 \mathrm{~cm}) \text { by US or LGND, } \\
\text { HGND proved by pathology (A1); }(2) \mathrm{HBV} \text { - or } \\
\text { HCV-related cirrhotic nodules (<1 cm) (B1); ( } 3 \text { ) } \\
\text { Synergistic risk factors such as HBV- or HCV- } \\
\text { related cirrhosis with diabetes or family history of } \\
\text { liver cancer without antiviral therapy or with LLV } \\
\text { after treatment (B1) }\end{array}$ & ND \\
\hline
\end{tabular}

SVR: Sustained virologic response; HBV: hepatitis B virus; HCV: hepatitis C virus; ALT: alanine aminotransferase; HBsAg: hepatitis B surface antigen; LLV: low-level viremia; THRI: Toronto Hepatocellular Carcinoma Risk Index; LGDN: low-grade dysplastic nodule; HGDN: high-grade dysplastic nodule; ND: no data available.

enhanced heterogeneity of an arterial phase, reduced tumor enhancement of the portal venous phase and/ or parenchymal equilibrium phase, and the enhancement patterns of rapid wash-in and wash-out are CT image features of liver cancer ${ }^{[84]}$. MRI has high tissue resolution, and enhanced scanning with gadolinium ethoxybenzyl diethylenetriamine pentaacetic acid (Gd-EOB-DTPA), a specific contrast agent, which can significantly improve the diagnostic sensitivity and specificity of early liver cancer ${ }^{[90]}$. The sensitivity and specificity of Gd-EOB-DTPA-enhanced MRI is $96.0 \%$ and $96.6 \%$, respectively, for cirrhotic nodules $\leq$ $2 \mathrm{~cm}$. EnhancedMRI has high sensitivity (94.7\%) and specificity (99.3\%) for HGDN and can accurately differentiate early HCC from HGDN ${ }^{[91]}$. A prospective monitoring (every 4-6 months) of 407 cirrhosis cases showed that among 43 patients diagnosed with liver cancer, the detection rate by enhanced MRI was $86 \%$, significantly higher than that by US $(27.9 \%)$. Moreover, the proportion of false positives was significantly lower than that by US (3.0\% and 5.6\%, respectively); $74.4 \%(32 / 43)$ of patients in this study had pre-early liver cancer (single nodule $<2 \mathrm{~cm}$ ) ${ }^{[92]}$. Therefore, compared with US, Gd-EOB-DTPA-enhanced MRI can improve the detection rate of pre-early HCC in cirrhotic patients who are at extremely high risk of $\mathrm{HCC}^{[90]}$. However, for liver tumors $\geq 2 \mathrm{~cm}$ in diameter, the sensitivity of CT and MRI is similar ${ }^{[87]}$. Whether enhanced MRI can replace US as a surveillance tool for a population with extremely high risk of liver cancer needs further studies.

\section{AFP, DCP, and AFP-L3}

In the 1970s, the sensitivity of AFP could reach $72 \%-87 \%$ due to the large proportion of moderate and advanced HCC. However, with the development of medical imaging and the increasing proportion of diagnosed early liver cancer, the value of AFP in the diagnosis of HCC has also decreased. The AFP level is related to tumor size, and serum AFP is elevated in some patients with benign liver disease, hepatoblastoma, and gastrointestinal malignancies ${ }^{[93]}$. Among liver cancers with tumors $<2 \mathrm{~cm}, 2-5 \mathrm{~cm}$, and $>5 \mathrm{~cm}$ in diameter, the proportion of normal AFP $(<20 \mathrm{ng} / \mathrm{mL})$ is $50 \%-70 \%, 30 \%-50 \%$, and $20 \%-30 \%$, 
respectively ${ }^{[87]}$. Overall, AFP has a sensitivity of $25 \%-65 \%$ and a specificity of $80 \%-94 \%$ for the diagnosis of $\mathrm{HCC}^{[87]}$. Therefore, AASLD 2010 and EASL 2018 no longer consider AFP as an essential indicator for the diagnosis of HCC. However, the combination of AFP and US can improve the sensitivity of diagnosis. A meta-analysis of 32 studies (13,367 cases) indicated that US alone had a lower sensitivity to detect HCC than when combined with AFP (RR: 0.88, 95\%CI: 0.83-0.93) ${ }^{[16]}$. A study of 1,487 HBV-infected patients showed that AFP alone could detect early HCC and the survival of patients could be prolonged; as such, AFP was remarked as a significant marker for HCC surveillance in areas with limited ultrasound equipment or detection experience ${ }^{[94]}$. At the same time, in this era of antiviral therapy, AFP can serve as an early detector of HBV-related HCC; portending the risk of HCC if antiviral therapy fails to decrease AFP levels ${ }^{[95]}$. Therefore, US combined with AFP can improve the sensitivity of early HCC, especially in patients with cirrhosis associated with non-viral hepatitis ${ }^{[96]}$.

DCP, also known as protein induced by vitamin $\mathrm{K}$ absence or antagonist II (PIVKA-II), was first reported to increase in the serum of patients with HCC in 1984. A meta-analysis of 31 studies showed that at a critical value of $40 \mathrm{mAU} / \mathrm{ml}$, the sensitivity and specificity of PIVKA-II in the diagnosis of HCC was 66\% and $89 \%$, respectively ${ }^{[97]}$. PIVKA-II is superior to AFP in the diagnosis of HCC irrespective of tumor size, population, and etiology. A multicenter study in China also showed that PIVKA-II was $6.2 \%-20.7 \%$ more accurate than AFP in distinguishing HCC from controls ${ }^{[98]}$. The formation mechanisms of PIVKA-II and AFP are different, and there was no correlation between them. In HCC patients who are AFP negative, the AUROC of PIVKA-II is 0.86. Therefore, the combination of AFP and PIVKA-II can improve the detection rate of early HCC.

AFP-L3 is a fucosylated variant of AFP, and the main component of AFP is AFP-L1 in patients with chronic hepatitis and cirrhosis, and AFP-L3 in patients with liver cancer. A meta-analysis showed that the overall sensitivity and specificity of AFP-L3 in the diagnosis of HCC was $48.3 \%$ (45.9\%-50.7\%) and $92.9 \%$ (91.6\%$94.0 \%{ }^{[99]}$. The GALAD model constructed based on gender, age, and three serum markers, AFP, AFP-L3, and DCP, had a sensitivity and specificity of $85.6 \%$ and $93.3 \%$, respectively, for the diagnosis of early liver cancer. It also had an AUROC greater than 0.9 for distinguishing liver cancer from chronic liver disease, and this model remained affected by etiology and antiviral therapy ${ }^{[100,101]}$.

\section{Other serum protein biomarkers}

HCC protein markers also include $\alpha$-L-fucosidase, serum ferritin, osteopontin, Glypican-3, heat shock protein 90 $\alpha$, Golgi protein 73, heat shock protein 27 and Dickkopf1. Since these biomarkers also increase in patients with other malignancies, cirrhosis, or chronic hepatitis, their value as liver cancer markers is limited $^{[102]}$

\section{New serum biomarkers}

\section{MicroRNA}

MiRNAs are non-coding RNAs consisting of 20-25 nucleotides that regulate the expression of target genes at the post-transcriptional level. MiRNAs encapsulated by exosomes are highly stable in the blood circulation and have prospects for use as serological markers. A recent meta-analysis showed that single miRNAs with $>80 \%$ sensitivity and specificity for diagnosing HCC include miR-130b, miR-150, miR-182, miR-215, and miR-96 ${ }^{[103]}$. Especially important was the finding that the diagnostic sensitivity and specificity of miRNA panel detection can reach $86.1 \%$ and $76.8 \%$, respectively, in pre-early liver cancer at BCLC stage $0^{[103]}$. Liver cancer diagnostic kits consisting of 7 miRNAs (miR-122, miR-192, miR-21, miR-223, miR-26a, miR-27a, and miR-801) have an accuracy of $94.1 \%$ in distinguishing liver cancer patients from normal people ${ }^{[104]}$. As a new marker, the main problem of miRNAs is suboptimal reproducibility. Its application value remains to be validated by large sample size, multicenter clinical trials. 


\section{Liquid biopsy}

Liquid biopsy as a marker for liver cancer detection in the era of precision medicine, includes circulating tumor cells, circulating tumor DNA (ctDNA), exosomes, tumor-associated methylation, circulating cellfree DNA (cfDNA), and long-stranded non-coding RNA, etc. ${ }^{[105,106]}$. Alteration in ctDNA-associated genes such as p15, p16, APC, SPINT2, SFRP1, p16INK4a, TFPI2, GSTP1, and RASSF1A, are related to development and progression of HCC. Studies have shown that cfDNA methylation, with high sensitivity and specificity in the diagnosis of liver cancer, is closely related to tumor size, tumor stage, and treatment response ${ }^{[107]}$. At present, the cost of liquid biopsy is high, not suitable for the screening and surveillance of liver cancer.

In summary, in addition to AFP, all new markers, including AFP-L3 and PIVKA II, as well as other serum protein markers and liquid biopsies, require adequate assessment by five clinical stages as proposed by EDRN for tumor marker discovery and validation ${ }^{[108]}$ so that they can eventually be used as indicators for liver cancer screening, surveillance, or diagnosis.

Recommendation 9: US with serum AFP is a first-line tool for liver cancer surveillance (A1).

Recommendation 10: Serum AFP combined with AFP-L3 and PIVKA- II can improve the detection rate of early liver cancer (B2).

Recommendation 11: In the extremely high-risk population of liver cancer, Gd-EOB-DTPA-enhanced MRI can improve the differential ability for dysplastic nodules, LGND and HGND, and significantly improve the detection rate of pre-early liver cancer (B1).

Recommendation 12: Liquid biopsy and other new liver cancer serum markers, whether alone or in combination, still lack sufficient clinical evaluation and sound validation for use as serum indicators for screening and surveillance of liver cancer; as such, they are not currently recommended for routine screening and surveillance $(\mathrm{C} 1)$.

\section{SURVEILLANCE OF LIVER CANCER}

For people at risk for developing liver cancer, lifelong surveillance is helpful for an early diagnosis and treatment of liver cancer to reduce mortality rate. A meta-analysis of 47 studies (including 15,158 patients with liver cirrhosis) showed that surveillance significantly improved the diagnostic rate (OR: 2.08, 95\%CI: 1.80-2.37) and radical cure rate (OR: 2.24, 95\%CI: 1.99-2.52) of liver cancer, which increased the OS rate of patients (OR: 1.90, 95\%CI: 1.67-2.17). The 3-year cumulative survival rate was $50.8 \%$ in the surveillance group and $27.9 \%$ in the non-surveillance group ${ }^{[109]}$. Another randomized controlled cohort of $17,820 \mathrm{HBV}$ infected patients showed that the 1- and 2-year survival rates of liver cancer patients in the surveillance group (AFP + US every 6 months) were $88.1 \%$ and $77.5 \%$, respectively, while the 1 -year survival rate of liver cancer patients in the non-surveillance group was $0 \%{ }^{[110]}$. Therefore, scientific determination of a surveillance target population and a stratified surveillance regimen are the most critical links to improve the OS rate and cost-effectiveness of liver cancer patients.

\section{Target population}

The following conditions should be met to determine the surveillance target population of liver cancer: (1) The risk of HCC must be high enough in the target population: The risks for progression to liver cancer differs among patients with liver diseases of different etiologies; in the era of antiviral therapy, the risk of liver cancer varies greatly in different clinical stages of HBV or HCV infection. Regardless of the presence of cirrhosis, only HBV-infected patients with an annual risk of $>1.5 \%$ for progression to liver cancer, or patients with an incidence of $>0.2 \%$ for liver cancer, are suitable for surveillance; (2) The diagnostic rate of HCC in the surveillance target population must be higher than that of early HCC as determined by symptoms, signs, or incidental imaging; and (3) The long-term survival rate after liver cancer treatment must be higher in the surveillance target population than in the non-surveillance population ${ }^{[111,12]}$. In a 
prospective cohort study of 8,900 patients with chronic viral liver diseases (15\% with HBV infection, $80 \%$ with HCV infection, and 5\% with mixed infection), liver cancer surveillance was performed by US plus AFP every 6 months in a median follow-up period of 10-year: $8.6 \%$ of the total subjects, which included $47.3 \%$ of patients with cirrhosis, progressed to $\mathrm{HCC}^{[113]}$. In cirrhosis-absent patients with chronic HBV or HCV infection, the annual incidence of liver cancer was low; especially, the risk of HCC was reduced by $70 \%$ after effective antiviral therapy ${ }^{[113]}$, failing to reach the threshold for surveillance of liver cancer. However, in patients with HBV-related cirrhosis, the annual incidence of HCC after antiviral therapy remained $1.5 \%-2.5 \%{ }^{[82,114]}$. Singal et al. ${ }^{[115]}$ prospectively analyzed 446 patients with Child A/B liver cirrhosis who were monitored by US plus AFP every 6 months in a median follow-up period of 3.5-year. The annual incidence of liver cancer was $2.8 \%$, and the 3 - and 5 -year cumulative incidences were $5.7 \%$ and $9.1 \%$, respectively, among which, early liver cancer accounted for $73.2 \%$. Another multicenter study showed that, for patients with chronic hepatitis B who were given ETV or TDF treatment, the annual incidence of HCC was $0.49 \%$ and $0.47 \%$ in patients without cirrhosis and $3.22 \%$ and $1.57 \%$ in patients with cirrhosis, during the first five and second five years, respectively. The results indicated that the incidence of HCC remained above the threshold of surveillance in patients with HBV-related cirrhosis receiving antiviral therapy. However, surveillance did not seem necessary for non-cirrhotic HBV patients who acquired SVR after antiviral therapy ${ }^{[16]}$. Therefore, patients with cirrhosis are a high-risk population that require surveillance of liver cancer. However, no multicenter, large sample, randomized controlled study on stratified surveillance of liver cancer in patients with cirrhosis has been conducted so far.

Notably, decompensated cirrhosis (Child-Pugh B or C) is associated with a higher risk of HCC ${ }^{[117]}$. However, since these patients have liver failure or uncontrollable complications that is not receptive to radical treatment for liver cancer, the AASLD guideline recommends that surveillance of liver cancer is not necessary from a cost-effectiveness point of view, unless such patients can afford liver transplantation. However, with the improvement of comprehensive treatment techniques for liver cancer, it is still necessary to monitor liver cancer in patients with decompensated cirrhosis if early diagnosis and treatment can prolong the life span of patients by more than 3 months. For patients with decompensated HBV-related cirrhosis, cirrhosis reversal or "recompensation" may also occur after effective antiviral therapy, so dynamic surveillance of liver cancer is still important to reduce the overall mortality of liver disease ${ }^{[48,14]}$. Surveillance is also necessary in patients awaiting liver transplantation because liver cancer may alter the priority of waiting and prognosis.

\section{Surveillance interval}

Liver cancer surveillance intervals are not consistently recommended by various guidelines worldwide. The new version of AASLD, EASL, LI-RADS, NCCN, and APASL guidelines, as well as the Guidelines for Diagnosis and Treatment of Primary Liver Cancer issued by the National Health Commission in 2019 recommend that the liver cancer population be monitored at 6 months intervals. The Japanese Society of Hepatology stratified the population for liver cancer risk, with a surveillance at intervals of 3-4 months for the extremely high-risk population and at 6 months for the low-risk population. The most of the patients under surveillance every 4 months had pre-early liver cancer and received radical treatment ${ }^{[118]}$. Scholars compared the surveillance data every 4 months and 12 months, respectively, in patients with chronic hepatitis and thrombocytopenia, and the results showed that the 3-year cumulative incidence of HCC was not significantly different between the 2 groups. In another study, clinical data of 52,823 patients diagnosed with HCC were retrospectively studied and analyzed. US was performed at intervals of 6, 12, 24, and 36 months before diagnosis. Among the monitored and unmonitored groups, 24.3\% (95\%CI: 23.7\%-24.9\%), $26.9 \%$ (95\%CI: $25.7 \%-28.2 \%$ ), $22.9 \%$ (95\%CI: $21.8 \%-24.1 \%$ ), $21.3 \%$ (95\%CI: $19.9 \%-22.8 \%$ ), and $18.3 \%$ (95\%CI: $17.8 \%-18.8 \%$ ) received radical treatment at the respective time points. Overall, the proportion of patients who could receive radical treatment was still very low with a 6-month interval surveillance $\operatorname{program}^{[119]}$. In patients with decompensated HBV-related cirrhosis, liver cancer was monitored by US and 


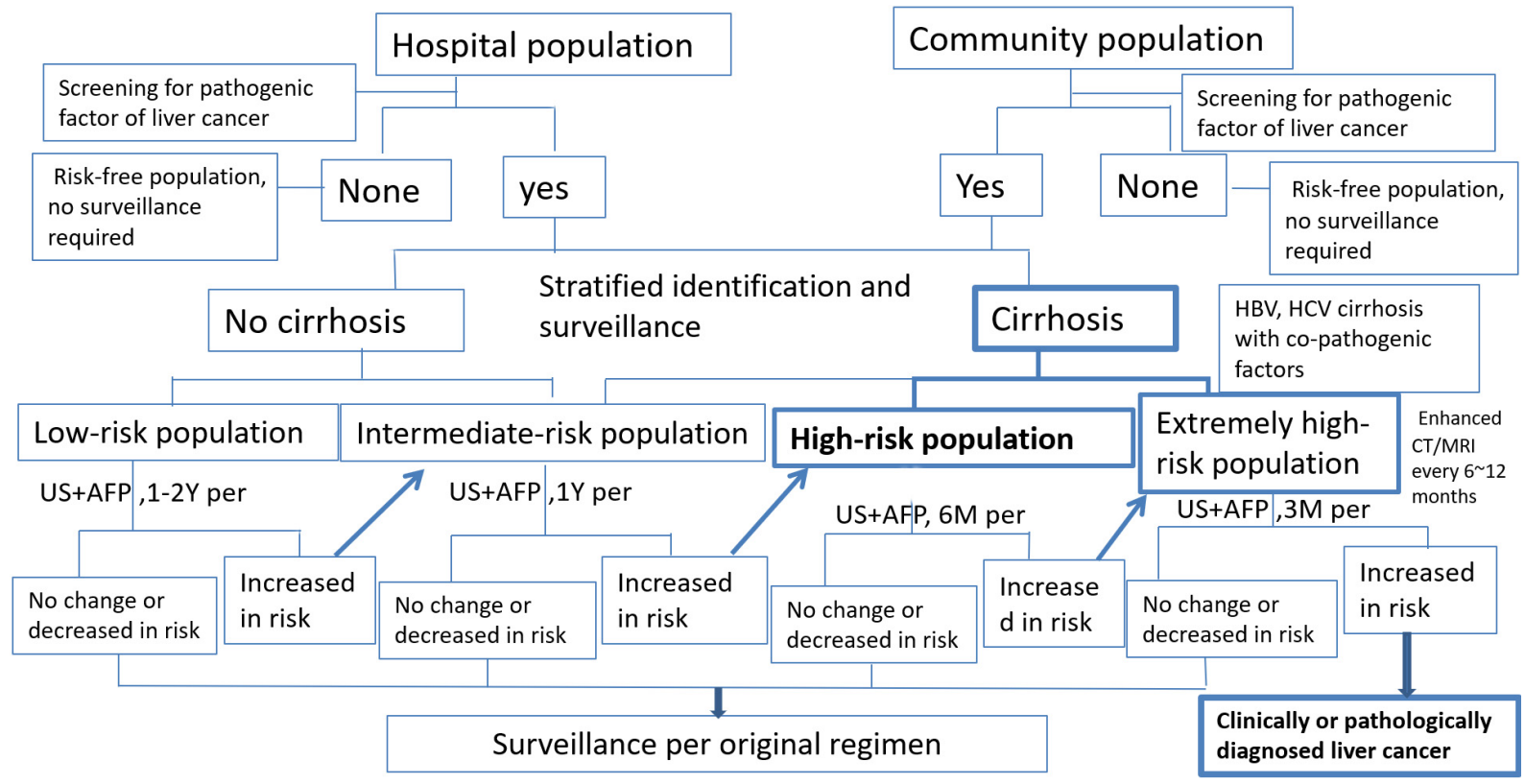

Figure 1. Map for stratified screening and surveillance of high-risk population of liver cancer. US: Abdominal ultrasound; AFP: alphafetoprotein.

AFP at 3 monthly intervals. In patients who developed liver cancer, $66.7 \%$ had BCLC stage A; while in the 6-month surveillance group, $15.4 \%$ of patients had BCLC stage A. $76.2 \%$ and $36.5 \%$ of patients in the 3 - and 6- monthly surveillance received radical treatment, respectively ${ }^{[120]}$. A multicenter randomized controlled study found that, among 1,278 patients with liver cirrhosis under surveillance for HCC at intervals of 3 and 6 months, 123 patients progressed to HCC. As the surveillance interval was shortened to 3 months, there was no difference in the diagnostic rate of early liver cancer $(<3 \mathrm{~cm}$ in diameter) $(79 \%$ and $70 \%)$ and the detection rate of focal nodules $(20.4 \%$ and $13.2 \%)$ between the two groups; however, the proportion of nodules $<1 \mathrm{~cm}$ was $41 \%$ in the 3 -month surveillance group, significantly higher than that in the 6-month surveillance group $(28 \%)^{[121]}$. Therefore, shortening the surveillance interval yields a higher detection rate of non-malignant lesions, but may also add unnecessary follow-ups. Based on community and hospital HBV or HCV infection cohorts, surveillance every 3-6 months for cirrhosis and every 6-12 months for chronic hepatitis can prolong the long-term survival of patients with liver cancer ${ }^{[122]}$. In areas with a high incidence of liver cancer, US surveillance at 2-year intervals is more cost-effective than a 2 -step screening process (the first step is screening for high-risk population of liver cancer and the second step is surveillance for liver cancer) in the general population over 50-year of age ${ }^{[123]}$.

Therefore, a scientific stratified management of high-risk population for liver cancer, and adoption of stratified surveillance intervals and surveillance tools are cost-effective options to address the incidence of HCC [Figure 1].

\section{Compliance for patients}

Previous studies have shown that the most important causes of moderate and advanced liver cancer are the absence of screening and surveillance, lack of interventions against liver cancer risk factors, and an obliviousness of underlying cirrhosis ${ }^{[124,125]}$. Overall, only $52 \%$ of patients had good compliance in clinical practice and were able to follow guidelines or expert suggestion for liver cancer screening and surveillance. In a multi-centre study that analyzed liver cancer surveillance compliance in 1,020 patients with cirrhosis, the results indicated that surveillance was not required for most patients if routine examination was 
normal or asymptomatic. $73.1 \%$ of patients received surveillance more than once, of these, $37.7 \%$ were under surveillance twice a year and were associated with hepatologists (OR: 30.1, 95\%CI: 17.5-51.8), while $50 \%$ reported difficulties with surveillance, including cost $(28.9 \%)$, time $(24.1 \%)$, and traffic $(17.8 \%)^{[126]}$. Similarly, in a cohort of chronic hepatitis $\mathrm{C}$ patients under long-term treatment, there was no screening in $13 \%$, no follow-up in $17 \%$ and no surveillance in $70 \%$ of patients who developed advanced liver cancer ${ }^{[127]}$. Therefore, early diagnosis of liver cancer depends on management of the whole course of treatment and a standardized surveillance program for patients at high risk.

\section{Recall}

Recall is the most important strategy to improve the early diagnosis rate during liver cancer surveillance. For nodules $\leq 1 \mathrm{~cm}$ detected by US, US examination can be repeated within 3-6 months. For 1-2 cm nodules detected by US, the use of Gd-EOB-DTPA-enhanced MRI examination can improve the identification of early liver cancer and $\mathrm{HGND}^{[90]}$. For indeterminate lesions, diagnostic liver biopsy is still required.

\section{Cost-effectiveness}

In high-risk populations for liver cancer, combined US and AFP surveillance is cost-effective ${ }^{[128]}$. Although early liver cancer can be detected by a 6-monthly US and/or AFP surveillance program in patients who are at low risk of liver cancer (annual incidence $<1 \%$ ), it is less cost-effective than in patients at high risk (annual incidence $>3 \%)^{[129]}$. Therefore, a stratified surveillance regimen is necessary for effective management of high-risk populations for liver cancer ${ }^{[130]}$. However, its cost-effectiveness still needs to be evaluated based on randomized, controlled, or large-sample cohort studies.

Recommendation 13: For patients with low risk of liver cancer, routine screening surveillance once every 1 year or more (C2); for patients with intermediate risk of liver cancer, routine surveillance once every 1 year (C1); for patients with high risk of liver cancer, routine surveillance once every 6 months (A1); for patients with extremely high risk of liver cancer, routine surveillance once every 3 months plus enhanced CT or MRI once every 6-12 months to improve the diagnostic rate of early liver cancer (B1).

Recommendation 14: Surveillance in patients with decompensated cirrhosis who face a higher risk of liver cancer might prolong survival, receive priority while waiting for liver transplantation and therefore improve prognosis, and reduce the overall mortality of cirrhosis (C2).

Recommendation 15: There are still some obstacles to liver cancer screening and surveillance. Science popularization, WeChat, or telephone visits can improve compliance with screening and surveillance in the whole-course management of chronic liver disease progressing to liver cancer (C1).

Recommendation 16: The stratified surveillance regimen for liver cancer can only show its costeffectiveness in the surveillance target population with adequate risk of liver cancer and adequate sample size $(\mathrm{C} 1)$.

\section{SURVEILLANCE AFTER RADICAL LIVER RESECTION}

Hepatectomy, liver transplantation, and local ablation therapy (radiofrequency ablation, microwave ablation, cryoablation and high-intensity focused ultrasound ablation, etc.) are the currently recommended guidelines for treatment of liver cancer and are listed as the most important radical methods for long survival of patients with liver cancer ${ }^{[5-10]}$. However, unfortunately, the 5 -year postoperative recurrence and metastasis rate of liver cancer is $40 \%-70 \%{ }^{[53,131-133]}$. Surveillance after radical resection of the liver is the most important measure for early detection of its recurrence and metastasis.

\section{Surveillance interval}

Studies have shown that the earliest recurrence of liver cancer can be 2 months after radical liver resection, and most patients have recurrence or metastasis 1-2 years after surgery ${ }^{[134]}$. There are many risk factors 
for recurrence and metastasis of liver cancer, including the presence of tiny, disseminated lesions before surgery, uneradicated multicenter hepatocarcinogenic factors of liver cancer, HBV mutation, and the patient's psychological and liver function status ${ }^{[132-137]}$. If recurrence of liver cancer is detected early, resection and local ablation can be re-performed to prolong the survival of patients and improve their quality of life. There is no consensus on liver cancer surveillance intervals after radical treatment, and appropriate surveillance tools and high-quality clinical evidence is also lacking.

\section{Prevention and treatment of recurrence and metastasis of liver cancer}

The prevention and treatment of recurrence and metastasis of liver cancer is still an unsolved clinical problem. High HBV DNA load and hepatic inflammation can lead to hepatocyte necrosis and regeneration of residual hepatocytes, resulting in a high recurrence rate of HBV-related liver cancer ${ }^{[131-133]}$. TDF has been shown to significantly reduce the risk of 5-year postoperative recurrence, liver transplantation, and death in patients with HBV-related liver cancer ${ }^{[138]}$. Therefore, domestic and international guidelines also recommend NAs to prevent and treat postoperative recurrence and metastasis in patients with HBV-related liver cancer ${ }^{[132]}$. There is insufficient evidence to support the role of antiangiogenic molecular targeted drugs in preventing recurrence of liver cancer after radical resection for advanced or relapsed liver cancer that does not meet the criteria for surgical resection. However, small molecule targeted drugs such as sorafenib, lenvatinib, and anlotinib, or combined therapies (such as immune checkpoint inhibitors combined with small molecule targeted drugs, and immune checkpoint inhibitors combined with anti-angiogenic targeted drugs) might be selected to prolong the overall survival time of patients ${ }^{[139,140]}$. For a detailed drug regimen, the reader is referred to the Guidelines for Diagnosis and Treatment of Primary Liver Cancer issued by the National Health Commission in 2019.

Recommendation 17: For patients who received radical treatment for liver cancer within 2-year, perform surveillance regimen that is prescribed for the population with extremely high risk of liver cancer, i.e., routine surveillance every 3 months, and enhanced CT or MRI to detect early recurrence and metastasis of liver cancer (C1). For excess of 2 -year, refer to the regimen for population with high risk of liver cancer, i.e., routine surveillance every 6 months $(\mathrm{C} 1)$.

Recommendation 18: Effective antiviral therapy can reduce the recurrence and metastasis of HBV-related liver cancer (B1). ETV, TDF, and TAF as first-line antiviral drugs for chronic hepatitis B can prevent and treat the recurrence and metastasis of HBV-related liver cancer (B1).

Recommendation 19: Antiangiogenic molecular targeted drugs alone or in combination with immune checkpoint inhibitors are used for the treatment of unresectable liver cancer (A1) as well as for the prevention of recurrence and metastasis of liver cancer after radical treatment (C2).

\section{ISSUES TO BE STUDIED AND ADDRESSED}

Evolution of precancerous diseases and precancerous lesions of liver cancer: There is a lack of specific biomarkers for detecting early liver cancer and progression through the clinical stages: from cirrhosis hepatic cirrhosis regenerative nodule - LGND - HGND - pre-early cancer - early cancer - to advanced liver cancer. Future studies are required to investigate the clinical value of AI-based medical imaging and identify big data markers in differentiating hepatic cirrhosis regenerative nodules from HGND and early liver cancer.

Surveillance intervals and appropriate tools for populations at different risk levels of liver cancer: One can utilize simple stratified identification models such as self-surveillance APP to detect high-risk population of liver cancer. However, randomized controlled or large-sample cohort study are still required to determine surveillance intervals of liver cancer; especially, high-quality clinical evidence is lacking to accurately determine surveillance intervals and tools for patients receiving radical resection of liver cancer. 
In the high-risk and extremely high-risk populations, health economic evaluation is needed to verify public health strategies and long-term cost-effectiveness of liver cancer surveillance.

Whether by medical staff or patients, compliance is still a major obstacle for liver cancer surveillance.

\section{DECLARATIONS}

\section{Authors' contributions}

Performed data acquisition, as well as provided administrative, technical, and material support: Ding $\mathrm{H}, \mathrm{Tu}$ H, Qu C, Cao G, Zhuang H, Zhao P, Xu X, Yang Y, Lu S

All authors contributed equally to conception, design and writing and performed data analysis and interpretation.

\section{Availability of data and materials}

Not applicable.

\section{Financial support and sponsorship}

This study was supported by the State Key Projects Specialized on Infectious Diseases (2017ZX10201201008, 2017ZX10203202-004, 2017ZX10201201-006, 2018ZX10725506, 2012ZX10002-008, 2010ZX10002008).

\section{Conflicts of interest}

All authors declared that there are no conflicts of interest.

\section{Ethical approval and consent to participate}

Not applicable.

\section{Consent for publication}

Not applicable.

\section{Copyright}

(c) The Author(s) 2021.

\section{REFERENCES}

1. Liver Cancer Professional Committee of Chinese Anti-Cancer Association, Liver Cancer Group, Chinese Association of Liver Diseases, Pathology Committee of Chinese Anti-Cancer Association. Evidence-based practice guidelines for the standardized pathological diagnosis of primary liver cancer (in Chinese, 2015 edition). J Clin Exp Pathol 2015:241-6.

2. Allemani C, Matsuda T, Di Carlo V, et al. Global surveillance of trends in cancer survival 2000-14 (CONCORD-3): analysis of individual records for 37513025 patients diagnosed with one of 18 cancers from 322 population-based registries in 71 countries. Lancet 2018;391:1023-75.

3. Tsilimigras DI, Bagante F, Sahara K, et al. Prognosis after resection of Barcelona Clinic Liver Cancer (BCLC) Stage 0, A, and B hepatocellular carcinoma: A comprehensive assessment of the current BCLC classification. Ann Surg Oncol 2019;26:3693-700.

4. Marrero JA, Kulik LM, Sirlin CB, et al. Diagnosis, staging, and management of hepatocellular carcinoma: 2018 Practice Guidance by the American Association for the Study of Liver Diseases. Hepatology 2018;68:723-50.

5. European Association for the Study of the Liver, European Association for the Study of the Liver. EASL Clinical Practice Guidelines: Management of hepatocellular carcinoma. J Hepatol 2018;69:182-236.

6. Korean Liver Cancer Association, National Cancer Center. 2018 Korean Liver Cancer Association-National Cancer Center Korea Practice Guidelines for the management of hepatocellular carcinoma. Gut Liver 2019;13:227-99.

7. Benson AB, D'Angelica MI, Abbott DE, et al. Guidelines insights: hepatobiliary cancers, version 2.2019. J Natl Compr Canc Netw 2019;17:302-10. 
8. Vogel A, Cervantes A, Chau I, et al; ESMO Guidelines Committee. Hepatocellular carcinoma: ESMO Clinical Practice Guidelines for diagnosis, treatment and follow-up. Ann Oncol 2018;29:iv238-55.

9. Omata M, Cheng AL, Kokudo N, et al. Asia-Pacific clinical practice guidelines on the management of hepatocellular carcinoma: a 2017 update. Hepatol Int 2017;11:317-70.

10. Department of Medical Administration, National Health and Health Commission of the People's Republic of China. [Guidelines for diagnosis and treatment of primary liver cancer in China (2019 edition)]. Zhonghua Gan Zang Bing Za Zhi 2020;28:112-28.

11. Kudo M, Matsui O, Izumi N, et al; Liver Cancer Study Group of Japan. JSH Consensus-Based Clinical Practice Guidelines for the management of hepatocellular carcinoma: 2014 update by the Liver Cancer Study Group of Japan. Liver Cancer 2014;3:458-68.

12. Kudo M. Management of hepatocellular carcinoma in Japan as a World-Leading Model. Liver Cancer 2018;7:134-47.

13. Yaman ME, Gudeloglu A, Senturk S, et al. A critical appraisal of the North American Spine Society guidelines with the Appraisal of Guidelines for Research and Evaluation II instrument. Spine $J$ 2015;15:777-81.

14. Kanwal F, Singal AG. Surveillance for hepatocellular carcinoma: current best practice and future direction. Gastroenterology 2019;157:54-64.

15. Tzartzeva K, Obi J, Rich NE, et al. Surveillance imaging and alpha fetoprotein for early detection of hepatocellular carcinoma in patients with cirrhosis: a meta-analysis. Gastroenterology 2018;154:1706-18.e1.

16. Atiq O, Tiro J, Yopp AC, et al. An assessment of benefits and harms of hepatocellular carcinoma surveillance in patients with cirrhosis. Hepatology 2017;65:1196-205.

17. Palmer WC, Patel T. Are common factors involved in the pathogenesis of primary liver cancers? A meta-analysis of risk factors for intrahepatic cholangiocarcinoma. J Hepatol 2012;57:69-76.

18. Wang M, Wang Y, Feng X, et al. Contribution of hepatitis B virus and hepatitis C virus to liver cancer in China north areas: Experience of the Chinese National Cancer Center. Int J Infect Dis 2017;65:15-21.

19. Expert consensus on multidisciplinary diagnosis and treatment of precancerous lesions of hepatocellular carcinoma (2020 edition). Zhonghua Gan Zang Bing Za Zhi 2020;28:14-20.

20. Nault JC, Martin Y, Caruso S, et al. Clinical impact of genomic diversity from early to advanced hepatocellular carcinoma. Hepatology 2020;71:164-82

21. Kobayashi M, Ikeda K, Hosaka T, et al. Dysplastic nodules frequently develop into hepatocellular carcinoma in patients with chronic viral hepatitis and cirrhosis. Cancer 2006;106:636-47.

22. Fateen W, Ryder SD. Screening for hepatocellular carcinoma: patient selection and perspectives. J Hepatocell Carcinoma 2017;4:71-9.

23. Zheng R, Qu C, Zhang S, et al. Liver cancer incidence and mortality in China: Temporal trends and projections to 2030. Chin J Cancer Res 2018;30:571-9.

24. Fitzmaurice C, Abate D, Abbasi N, et al; Global Burden of Disease Cancer Collaboration. Global, regional, and national cancer incidence, mortality, years of life lost, years lived with disability, and disability-adjusted life-years for 29 cancer groups, 1990 to 2017: a systematic analysis for the Global Burden of Disease Study. JAMA Oncol 2019;5:1749-68.

25. Prevention of Infection-related Cancer (PIRCA) Group, Specialized Commmittee of Cancer Prevention and Control, Chinese Preventive Medicine Association; Non-communicable \& Chronic Disease Control and Prevention Society, Chinese Preventive Medicine Association; Heallth Communication Society, Chinese Preventive Medicine Association. Strategies of primary prevention of liver cancer in China: expert consensus (2018). Chin J Oncol 2018,40:550-7.

26. Zeng HM, Cao MM, Zheng RS, et al. Trend analysis of age of diagnosis for liver cancer in cancer registry areas of China, 2000-2014. Zhonghua Yu Fang Yi Xue Za Zhi 2018;52:573-8.

27. Zhou M, Wang H, Zeng X, et al. Mortality, morbidity, and risk factors in China and its provinces, 1990-2017: a systematic analysis for the Global Burden of Disease Study 2017. Lancet 2019;394:1145-58.

28. Eslam M, Sanyal AJ, George J; International Consensus Panel. MAFLD: a consensus-driven proposed nomenclature for metabolic associated fatty liver disease. Gastroenterology 2020;158:1999-2014.e1.

29. Forner A, Reig M, Bruix J. Hepatocellular carcinoma. Lancet 2018;391:1301-14.

30. Chinese Society of Infectious Diseases, Chinese Medical Association., Chinese Society of Hepatology, Chinese Medical Association. The guidelines of prevention and treatment for chronic hepatitis B (2019 version). Zhonghua Gan Zang Bing Za Zhi 2019;27:938-61.

31. Chinese Society of Hepatology., Chinese Society of Infectious Diseases, Chinese Medical Association. [Guidelines for the prevention and treatment of hepatitis C (2019 version)]. Zhonghua Gan Zang Bing Za Zhi 2019;27:962-79.

32. Sharma SA, Kowgier M, Hansen BE, et al. Toronto HCC risk index: A validated scoring system to predict 10-year risk of HCC in patients with cirrhosis. J Hepatol 2017:92-9.

33. Ganne-Carrié N, Chaffaut C, Bourcier V, et al; for CIRRAL Group. Estimate of hepatocellular carcinoma incidence in patients with alcoholic cirrhosis. J Hepatol 2018;69:1274-83.

34. Kim BS, Seo YS, Kim YS, et al; Korean Transient Elastography Study Group. Reduced risk of hepatocellular carcinoma by achieving a subcirrhotic liver stiffness through antiviral agents in hepatitis B virus-related advanced fibrosis or cirrhosis. $J$ Gastroenterol Hepatol 2018;33:503-10.

35. de Martel C, Georges D, Bray F, Ferlay J, Clifford GM. Global burden of cancer attributable to infections in 2018: a worldwide incidence analysis. Lancet Global Health 2020;8:e180-90.

36. Shi Y, Wu YH, Wu W, Zhang WJ, Yang J, Chen Z. Association between occult hepatitis B infection and the risk of hepatocellular carcinoma: a meta-analysis. Liver Int 2012;32:231-40.

37. Loomba R, Liu J, Yang HI, et al; REVEAL-HBV Study Group. Synergistic effects of family history of hepatocellular carcinoma and 
hepatitis B virus infection on risk for incident hepatocellular carcinoma. Clin Gastroenterol Hepatol 2013;11:1636-45.e1-3.

38. Fan C, Li M, Gan Y, et al. A simple AGED score for risk classification of primary liver cancer: development and validation with longterm prospective HBsAg-positive cohorts in Qidong, China. Gut 2019;68:948-9.

39. Yang H, Yuen M, Chan HL, et al. Risk estimation for hepatocellular carcinoma in chronic hepatitis B (REACH-B): development and validation of a predictive score. Lancet Oncology 2011;12:568-74.

40. Tseng TC, Liu CJ, Hsu CY, et al. High level of hepatitis B core-related antigen associated with increased risk of hepatocellular carcinoma in patients with chronic HBV infection of intermediate viral load. Gastroenterology 2019;157:1518-1529.e3.

41. Tseng TC, Liu CJ, Yang HC, et al. High levels of hepatitis B surface antigen increase risk of hepatocellular carcinoma in patients with low HBV load. Gastroenterology 2012;142:1140-1149.e3; quiz e13-4.

42. Baudi I, Inoue T, Tanaka Y. Novel Biomarkers of Hepatitis B and Hepatocellular Carcinoma: Clinical Significance of HBcrAg and M2BPGi. Int J Mol Sci 2020;21:949.

43. Liu S, Zhang $\mathrm{H}, \mathrm{Gu} \mathrm{C}$, et al. Associations between hepatitis B virus mutations and the risk of hepatocellular carcinoma: a meta-analysis. $J$ Natl Cancer Inst 2009;101:1066-82.

44. Yin J, Xie J, Liu S, et al. Association between the various mutations in viral core promoter region to different stages of hepatitis B, ranging of asymptomatic carrier state to hepatocellular carcinoma. Am J Gastroenterol 2011;106:81-92.

45. Bai X, Zhu Y, Jin Y, et al. Temporal acquisition of sequential mutations in the enhancer II and basal core promoter of HBV in individuals at high risk for hepatocellular carcinoma. Carcinogenesis 2011;32:63-8.

46. Yin J, Xie J, Zhang H, et al. Significant association of different preS mutations with hepatitis B-related cirrhosis or hepatocellular carcinoma. J Gastroenterol 2010;45:1063-71.

47. Lin CL, Kao JH. Prevention of hepatitis B virus-related hepatocellular carcinoma. Hepatoma Res 2021;7:9.

48. Yip TC, Wong VW, Chan HL, Tse YK, Lui GC, Wong GL. Tenofovir is associated with lower risk of hepatocellular carcinoma than entecavir in patients with chronic HBV infection in China. Gastroenterology 2020;158:215-225.e6.

49. Yin J, Wang J, Pu R, et al. Hepatitis B virus combo mutations improve the prediction and active prophylaxis of hepatocellular carcinoma: a clinic-based cohort study. Cancer Prev Res (Phila) 2015;8:978-88.

50. Han Y, Zeng A, Liao H, Liu Y, Chen Y, Ding H. The efficacy and safety comparison between tenofovir and entecavir in treatment of chronic hepatitis B and HBV related cirrhosis: A systematic review and Meta-analysis. Int Immunopharmacol 2017;42:168-75.

51. Ren H, Huang Y. Effects of pegylated interferon- $\alpha$ based therapies on functional cure and the risk of hepatocellular carcinoma development in patients with chronic hepatitis B. J Viral Hepat 2019;26 Suppl 1:5-31.

52. Maucort-Boulch D, de Martel C, Franceschi S, Plummer M. Fraction and incidence of liver cancer attributable to hepatitis B and C viruses worldwide. Int J Cancer 2018;142:2471-7.

53. Yang F, Ma L, Yang Y, et al. Contribution of hepatitis B virus infection to the aggressiveness of primary liver cancer: a clinical epidemiological study in Eastern China. Front Oncol 2019;9:370.

54. Turati F, Edefonti V, Talamini R, et al. Family history of liver cancer and hepatocellular carcinoma. Hepatology 2012;55:1416-25.

55. Ma L, Liu J, Wang W, et al. Direct-acting antivirals and interferon-based therapy on hepatocellular carcinoma risk in chronic hepatitis-C patients. Future Oncol 2020;16:675-86.

56. Hassan MM, Hwang LY, Hatten CJ, et al. Risk factors for hepatocellular carcinoma: synergism of alcohol with viral hepatitis and diabetes mellitus. Hepatology 2002;36:1206-13.

57. Shen Y, Risch H, Lu L, et al. Risk factors for hepatocellular carcinoma (HCC) in the northeast of the United States: results of a casecontrol study. Cancer Causes Control 2020;31:321-32.

58. Galicia-Moreno M, Monroy-Ramirez HC, Campos-Valdez M, et al. Hepatocellular carcinoma and hepatitis C virus infection in Latin America: epidemiology, diagnosis and treatment. Hepatoma Res 2020;6:20.

59. Heckley GA, Jarl J, Asamoah BO, G-Gerdtham U. How the risk of liver cancer changes after alcohol cessation: a review and metaanalysis of the current literature. BMC Cancer 2011;11:446.

60. Berkan-Kawińska A, Piekarska A. Hepatocellular carcinoma in non-alcohol fatty liver disease - changing trends and specific challenges. Curr Med Res Opin 2020;36:235-43.

61. Nevola R, Rinaldi L, Giordano M, et al. Mechanisms and clinical behavior of hepatocellular carcinoma in HBV and HCV infection and alcoholic and non-alcoholic fatty liver disease. Hepatoma Res 2018;4:55.

62. Alexander M, Loomis AK, van der Lei J, et al. Risks and clinical predictors of cirrhosis and hepatocellular carcinoma diagnoses in adults with diagnosed NAFLD: real-world study of 18 million patients in four European cohorts. BMC Med 2019;17:95.

63. Wang Z, Zhao X, Chen S, et al. Associations between nonalcoholic fatty liver disease and cancers in a large cohort in China. Clin Gastroenterol Hepatol 2020:S1542-3565(20)30643-1.

64. Yang WS, Va P, Bray F, et al. The role of pre-existing diabetes mellitus on hepatocellular carcinoma occurrence and prognosis: a metaanalysis of prospective cohort studies. PLoS One 2011;6:e27326.

65. Pan XF, He M, Yu C, et al; China Kadoorie Biobank Collaborative Group. Type 2 diabetes and risk of incident cancer in China: a prospective study among 0.5 million Chinese adults. Am J Epidemiol 2018;187:1380-91.

66. Ross RK, Yuan JM, Yu MC, et al. Urinary aflatoxin biomarkers and risk of hepatocellular carcinoma. Lancet 1992;339:943-6.

67. Sun Z, Chen T, Thorgeirsson SS, et al. Dramatic reduction of liver cancer incidence in young adults: 28 year follow-up of etiological interventions in an endemic area of China. Carcinogenesis 2013;34:1800-5.

68. Ng AWT, Poon SL, Huang MN, et al. Aristolochic acids and their derivatives are widely implicated in liver cancers in Taiwan and throughout Asia. Sci Transl Med 2017;9:eaan6446. 
69. Chen CJ, Yang YH, Lin MH, et al; Health Data Analysis in Taiwan (hDATa) Research Group. Herbal medicine containing aristolochic acid and the risk of hepatocellular carcinoma in patients with hepatitis B virus infection. Int J Cancer 2018;143:1578-87.

70. Chiang CJ, Yang YW, You SL, Lai MS, Chen CJ. Thirty-year outcomes of the national hepatitis B immunization program in Taiwan. JAMA 2013;310:974-6.

71. Thomas DL. Global Elimination of Chronic Hepatitis. N Engl J Med 2019;380:2041-50.

72. Zhang H, Zhu J, Xi L, Xu C, Wu A. Validation of the Toronto hepatocellular carcinoma risk index for patients with cirrhosis in China: a retrospective cohort study. World J Surg Oncol 2019;17:75.

73. Dyson J, Jaques B, Chattopadyhay D, et al. Hepatocellular cancer: the impact of obesity, type 2 diabetes and a multidisciplinary team. $J$ Hepatol 2014;60:110-7.

74. Kim JH, Sinn DH, Kang W, et al. Low-level viremia and the increased risk of hepatocellular carcinoma in patients receiving entecavir treatment. Hepatology 2017;66:335-43.

75. Wang HM, Hung $\mathrm{CH}, \mathrm{Lu} \mathrm{SN}$, et al. Liver stiffness measurement as an alternative to fibrotic stage in risk assessment of hepatocellular carcinoma incidence for chronic hepatitis C patients. Liver Int 2013;33:756-61.

76. Lee HA, Lee HW, Kim IH, et al. Extremely low risk of hepatocellular carcinoma development in patients with chronic hepatitis B in immune-tolerant phase. Aliment Pharmacol Ther 2020;52:196-204.

77. Papatheodoridis G, Dalekos G, Sypsa V, et al. PAGE-B predicts the risk of developing hepatocellular carcinoma in Caucasians with chronic hepatitis B on 5-year antiviral therapy. J Hepatol 2016;64:800-6.

78. Yip TC, Wong GL, Wong VW, et al. Reassessing the accuracy of PAGE-B-related scores to predict hepatocellular carcinoma development in patients with chronic hepatitis B. J Hepatol 2020;72:847-54.

79. Papatheodoridis GV, Sypsa V, Dalekos GN, et al. Hepatocellular carcinoma prediction beyond year 5 of oral therapy in a large cohort of Caucasian patients with chronic hepatitis B. J Hepatol 2020;72:1088-96.

80. Li L, Liu W, Chen YH, et al. Antiviral drug resistance increases hepatocellular carcinoma: a prospective decompensated cirrhosis cohort study. World J Gastroenterol 2013;19:8373-81.

81. Raimondi S, Bruno S, Mondelli MU, Maisonneuve P. Hepatitis C virus genotype $1 \mathrm{~b}$ as a risk factor for hepatocellular carcinoma development: a meta-analysis. J Hepatol 2009;50:1142-54.

82. Pons M, Rodríguez-Tajes S, Esteban JI, et al. Non-invasive prediction of liver-related events in patients with HCV-associated compensated advanced chronic liver disease after oral antivirals. J Hepatol 2020;72:472-80.

83. Younossi ZM. Non-alcoholic fatty liver disease - A global public health perspective. J Hepatol 2019;70:531-44.

84. Liu X, Baecker A, Wu M, et al. Family history of liver cancer may modify the association between HBV infection and liver cancer in a Chinese population. Liver Int 2019;39:1490-503.

85. Fan R, Papatheodoridis G, Sun J, et al. aMAP risk score predicts hepatocellular carcinoma development in patients with chronic hepatitis. J Hepatol 2020;73:1368-78.

86. Erkan B, Meier J, Clark TJ, Kaplan J, Lambert JR, Chang S. Non-invasive diagnostic criteria of hepatocellular carcinoma: Comparison of diagnostic accuracy of updated LI-RADS with clinical practice guidelines of OPTN-UNOS, AASLD, NCCN, EASL-EORTC, and KLSCG-NCC. PLoS One 2019;14:e226291.

87. Harris PS, Hansen RM, Gray ME, Massoud OI, McGuire BM, Shoreibah MG. Hepatocellular carcinoma surveillance: An evidence-based approach. World J Gastroenterol 2019;25:1550-9.

88. Kudo M, Ueshima K, Osaki Y, et al. B-Mode ultrasonography versus contrast-enhanced ultrasonography for surveillance of hepatocellular carcinoma: a prospective multicenter randomized controlled trial. Liver Cancer 2019;8:271-80.

89. Zhang J, Yu Y, Li Y, Wei L. Diagnostic value of contrast-enhanced ultrasound in hepatocellular carcinoma: a meta-analysis with evidence from 1998 to 2016. Oncotarget 2017;8:75418-26.

90. Kim TH, Yoon JH, Lee JM. Emerging role of hepatobiliary magnetic resonance contrast Media and contrast-enhanced ultrasound for noninvasive diagnosis of hepatocellular carcinoma: emphasis on recent updates in Major Guidelines. Korean J Radiol 2019;20:863-79.

91. Renzulli M, Biselli M, Brocchi S, et al. New hallmark of hepatocellular carcinoma, early hepatocellular carcinoma and high-grade dysplastic nodules on Gd-EOB-DTPA MRI in patients with cirrhosis: a new diagnostic algorithm. Gut 2018;67:1674-82.

92. Kim SY, An J, Lim YS, et al. MRI with liver-specific contrast for surveillance of patients with cirrhosis at high risk of hepatocellular carcinoma. JAMA Oncol 2017;3:456-63.

93. Zhang X, Wang T, Zhang KH, Chen SH, He YT, Wang YQ. Simple clinical metrics enhance AFP to effectively identify cirrhotic patients with complicating hepatocellular carcinoma at various AFP levels. Front Oncol 2019;9:1478.

94. McMahon BJ, Bulkow L, Harpster A, et al. Screening for hepatocellular carcinoma in Alaska natives infected with chronic hepatitis B: a 16-year population-based study. Hepatology 2000;32:842-6.

95. Wong GL, Chan HL, Tse YK, et al. On-treatment alpha-fetoprotein is a specific tumor marker for hepatocellular carcinoma in patients with chronic hepatitis B receiving entecavir. Hepatology 2014;59:986-95.

96. Worland T, Harrison B, Delmenico L, Dowling D. Hepatocellular carcinoma screening utilising serum alpha-fetoprotein measurement and abdominal ultrasound is more effective than ultrasound alone in patients with non-viral cirrhosis. J Gastrointest Cancer 2018;49:47680.

97. Xing H, Zheng YJ, Han J, et al. Protein induced by vitamin K absence or antagonist-II versus alpha-fetoprotein in the diagnosis of hepatocellular carcinoma: A systematic review with meta-analysis. Hepatobiliary Pancreat Dis Int 2018;17:487-95.

98. Ji J, Wang H, Li Y, et al. Diagnostic evaluation of des-gamma-carboxy prothrombin versus $\alpha$-fetoprotein for hepatitis B virus-related hepatocellular carcinoma in China: A large-scale, multicentre study. PLoS One 2016;11:e0153227. 
99. Yi X, Yu S, Bao Y. Alpha-fetoprotein-L3 in hepatocellular carcinoma: a meta-analysis. Clin Chim Acta 2013;425:212-20.

100. Best J, Bilgi H, Heider D, et al. The GALAD scoring algorithm based on AFP, AFP-L3, and DCP significantly improves detection of BCLC early stage hepatocellular carcinoma. Z Gastroenterol 2016;54:1296-305.

101. Berhane S, Toyoda H, Tada T, et al. Role of the GALAD and BALAD-2 serologic models in diagnosis of hepatocellular carcinoma and prediction of survival in patients. Clin Gastroenterol Hepatol 2016;14:875-886.e6.

102. Chen H, Zhang Y, Li S, et al. Direct comparison of five serum biomarkers in early diagnosis of hepatocellular carcinoma. Cancer Manag Res 2018;10:1947-58.

103. Peng C, Ye Y, Wang Z, et al. Circulating microRNAs for the diagnosis of hepatocellular carcinoma. Dig Liver Dis 2019;51:621-31.

104. Zhou J, Yu L, Gao X, et al. Plasma microRNA panel to diagnose hepatitis B virus-related hepatocellular carcinoma. J Clin Oncol 2011;29:4781-8.

105. Wan JCM, Massie C, Garcia-Corbacho J, et al. Liquid biopsies come of age: towards implementation of circulating tumour DNA. Nat Rev Cancer 2017;17:223-38.

106. Cai J, Chen L, Zhang Z, et al. Genome-wide mapping of 5-hydroxymethylcytosines in circulating cell-free DNA as a non-invasive approach for early detection of hepatocellular carcinoma. Gut 2019;68:2195-205.

107. Xu RH, Wei W, Krawczyk M, et al. Circulating tumour DNA methylation markers for diagnosis and prognosis of hepatocellular carcinoma. Nat Mater 2017;16:1155-61.

108. Prensner JR, Chinnaiyan AM, Srivastava S. Systematic, evidence-based discovery of biomarkers at the NCI. Clin Exp Metastasis 2012;29:645-52.

109. Singal AG, Pillai A, Tiro J. Early detection, curative treatment, and survival rates for hepatocellular carcinoma surveillance in patients with cirrhosis: a meta-analysis. PLoS Med 2014;11:e1001624.

110. Yang B, Zhang B, Xu Y, et al. Prospective study of early detection for primary liver cancer. J Cancer Res Clin Oncol 1997;123:357-60.

111. Giannini EG, Cucchetti A, Erroi V, Garuti F, Odaldi F, Trevisani F. Surveillance for early diagnosis of hepatocellular carcinoma: how best to do it? World J Gastroenterol 2013;19:8808-21.

112. Frenette CT, Isaacson AJ, Bargellini I, Saab S, Singal AG. A Practical Guideline for hepatocellular carcinoma screening in patients at risk. Mayo Clin Proc Innov Qual Outcomes 2019;3:302-10.

113. Izzo F, Piccirillo M, Albino V, et al. Prospective screening increases the detection of potentially curable hepatocellular carcinoma: results in 8,900 high-risk patients. HPB (Oxford) 2013;15:985-90.

114. Lok AS, McMahon BJ, Brown RS Jr, et al. Antiviral therapy for chronic hepatitis B viral infection in adults: A systematic review and meta-analysis. Hepatology 2016;63:284-306.

115. Singal AG, Conjeevaram HS, Volk ML, et al. Effectiveness of hepatocellular carcinoma surveillance in patients with cirrhosis. Cancer Epidemiol Biomarkers Prev 2012;21:793-9.

116. Papatheodoridis GV, Idilman R, Dalekos GN, et al. The risk of hepatocellular carcinoma decreases after the first 5 years of entecavir or tenofovir in Caucasians with chronic hepatitis B. Hepatology 2017;66:1444-53.

117. Jepsen P, Kraglund F, West J, Villadsen GE, Sørensen HT, Vilstrup H. Risk of hepatocellular carcinoma in Danish outpatients with alcohol-related cirrhosis. J Hepatol 2020;73:1030-6.

118. Wang JH, Chang KC, Kee KM, et al. Hepatocellular carcinoma surveillance at 4- vs. 12-month intervals for patients with chronic viral hepatitis: a randomized study in community. Am J Gastroenterol 2013;108:416-24.

119. Wu CY, Hsu YC, Ho HJ, Chen YJ, Lee TY, Lin JT. Association between ultrasonography screening and mortality in patients with hepatocellular carcinoma: a nationwide cohort study. Gut 2016;65:693-701.

120. Li P, Ding HG, Xu H, et al. Shorten liver cancer surveillance interval may improve the prognosis of patients with hepatitis B cirrhosisrelated hepatocellular carcinoma. J Clin Hepatol 2017;33:1301-4.

121. Trinchet JC, Chaffaut C, Bourcier V, et al; Groupe d'Etude et de Traitement du Carcinome Hépatocellulaire (GRETCH). Ultrasonographic surveillance of hepatocellular carcinoma in cirrhosis: a randomized trial comparing 3- and 6-month periodicities. Hepatology 2011;54:1987-97.

122. Kee KM, Lu SN. Hospital- and community-based screenings for hepatocellular carcinoma in Taiwan. Oncology 2011;81 Suppl 1:36-40.

123. Kuo MJ, Chen HH, Chen CL, et al. Cost-effectiveness analysis of population-based screening of hepatocellular carcinoma: Comparing ultrasonography with two-stage screening. World J Gastroenterol 2016;22:3460-70.

124. Goldberg DS, Taddei TH, Serper M, et al. Identifying barriers to hepatocellular carcinoma surveillance in a national sample of patients with cirrhosis. Hepatology 2017;65:864-74.

125. Zhao C, Jin M, Le RH, et al. Poor adherence to hepatocellular carcinoma surveillance: A systematic review and meta-analysis of a complex issue. Liver Int 2018;38:503-14.

126. Singal AG, Tiro JA, Murphy CC, et al. Patient-reported barriers are associated with receipt of hepatocellular carcinoma surveillance in a multicenter cohort of patients with cirrhosis. Clin Gastroenterol Hepatol 2020:S1542-3565(20)30912-5.

127. Singal AG, Tiro J, Li X, Adams-Huet B, Chubak J. Hepatocellular carcinoma surveillance among patients with cirrhosis in a populationbased integrated Health Care Delivery System. J Clin Gastroenterol 2017;51:650-5.

128. Likhitsup A, Parikh ND. Economic implications of hepatocellular carcinoma surveillance and treatment: A guide for clinicians. Pharmacoeconomics 2020;38:5-24.

129. Goossens N, Singal AG, King LY, et al. Cost-effectiveness of risk score-stratified hepatocellular carcinoma screening in patients with cirrhosis. Clin Transl Gastroenterol 2017;8:e101.

130. Lu WP, Dong JH. Hepatectomy for hepatocellular carcinoma in the era of liver transplantation. World J Gastroenterol 2014;20:9237-44. 
131. Yin J, Li N, Han Y, et al. Effect of antiviral treatment with nucleotide/nucleoside analogs on postoperative prognosis of hepatitis B virusrelated hepatocellular carcinoma: a two-stage longitudinal clinical study. J Clin Oncol 2013;31:3647-55.

132. Chen L, Zhang Q, Chang W, et al. Viral and host inflammation-related factors that can predict the prognosis of hepatocellular carcinoma. Eur J Cancer 2012;48:1977-87.

133. Lu WP, Dong JH. Hepatectomy for hepatocellular carcinoma in the era of liver transplantation. World J Gastroenterol 2014;20:9237-44.

134. Chen Q, Shu C, Laurence AD, et al. Effect of Huaier granule on recurrence after curative resection of HCC: a multicentre, randomised clinical trial. Gut 2018;67:2006-16.

135. Saito R, Amemiya H, Hosomura N, et al. Prognostic factors for post-recurrent survival in hepatocellular carcinoma after curative resection. Anticancer Res 2019;39:3033-8.

136. Zhou HY, Luo Y, Chen WD, et al. Hepatitis B virus mutation may play a role in hepatocellular carcinoma recurrence: A systematic review and meta-regression analysis. J Gastroenterol Hepatol 2015;30:977-983.

137. Yin J, Chen X, Li N, et al. Compartmentalized evolution of hepatitis B virus contributes differently to the prognosis of hepatocellular carcinoma. Carcinogenesis 2020:bgaa127.

138. Choi J, Jo C, Lim YS. Tenofovir Versus Entecavir on Recurrence of Hepatitis B Virus-Related Hepatocellular Carcinoma After Surgical Resection. Hepatology 2020.

139. Finn RS, Qin S, Ikeda M, et al; IMbrave150 Investigators. Atezolizumab plus Bevacizumab in unresectable hepatocellular carcinoma. $N$ Engl J Med 2020;382:1894-905.

140. He C, Wu T, Hao Y. Anlotinib induces hepatocellular carcinoma apoptosis and inhibits proliferation via Erk and Akt pathway. Biochem Biophys Res Commun 2018;503:3093-9. 